\title{
A new sample of $X$-ray selected narrow emission-line galaxies
}

\section{The nature of optically elusive AGN}

\author{
E. Pons and M. G. Watson \\ Department of Physics \& Astronomy, University of Leicester, Leicester, LE1 7RH, UK \\ Received 21 March 2014 / Accepted 15 July 2014

\begin{abstract}
Using the 3XMM catalogue of serendipitous X-ray sources, and the SDSS-DR9 spectroscopic catalogue, we have obtained a new sample of X-ray selected narrow emission line galaxies. The standard optical diagnostic diagram and selection by hard X-ray luminosity expose a mismatch between the optically-based and X-ray-based classifications. The nature of these misclassified elusive AGN can be understood in terms of their broader X-ray and optical properties and leads to a division of this sub-sample into two groups. A little more than half are likely to be narrow-line Seyfert 1s (NLS1s), so misclassified because of the contribution of the broad line region to their optical spectra. The remainder have some of the properties of Seyfert 2 (Sy2) AGN; their optical elusiveness can be explained by optical dilution from the host galaxy plus a star-formation contribution and by their underluminous optical emission due to low accretion rates. Because some of the Sy2 sources have very low accretion rates, are unabsorbed, plus the fact that they lack broad optical emission lines, they are good candidates to be true Sy2 AGN.
\end{abstract}

Key words. galaxies: active - galaxies: Seyfert $-\mathrm{X}$-rays: galaxies

\section{Introduction}

There is strong observational evidence that most, if not all, massive galaxies $\left(M_{*}>10^{10}-10^{12} M_{\odot}\right)$ in the nearby Universe host a central supermassive black hole (SMBH; Kormendy \& Richstone 1995). Central black holes are less common in lowmass systems, but have still been identified in some low mass and dwarf galaxies (Filippenko \& Ho 2003; Barth et al. 2004; Reines et al. 2011). The central sources in galaxies are observed as active galactic nuclei (AGN) as the SMBH grows through a phase of significant mass accretion.

With large surveys at different wavelengths, various criteria can be used to identify AGN. In the optical, type 2 AGN can be distinguished from star-forming (SF) galaxies on the basis of their emission lines. The most commonly used diagnostic for large sample of galaxies is the BPT diagram introduced by Baldwin et al. (1981). This considers the emission-line ratios to probe the source of excitation. In the case of photoionization from young and massive $\mathrm{O}$ stars (in star-forming galaxies), the low ionization transitions (i.e. the collisionally-excited lines) $[\mathrm{NII}],[\mathrm{SII}],[\mathrm{OIII}]$ and especially $[\mathrm{OI}]$ are very weak relative to the Balmer recombination lines. In contrast, for photoionization from an accretion disc around a SMBH, the collisionally-excited lines will be stronger because the photons from an AGN extend to higher energy.

However, some X-ray selected AGN, selected by their hard X-ray luminosity, will not be classified as AGN by their optical emission line properties. This class of sources has been given a variety of names such as elusive $\mathrm{AGN}^{1}$ (Caccianiga et al. 2007), optically dull AGN (Elvis et al. 1981;

\footnotetext{
1 In this paper, the term "elusive" AGN refers to objects with X-ray emission like an AGN but optical emission line ratios like a star-forming galaxy.
}

Trump et al. 2009) or also X-ray bright, optically normal galaxies (XBONG; Comastri et al. 2002). Such galaxies are particularly interesting because, despite their luminous X-ray emission, they lack clear optical signatures of an AGN. Previous studies have shown that many of these sources are not so special but are indeed normal type 2 AGN diluted by a bright host (Moran et al. 2002; Caccianiga et al. 2007); however about 10-20\% are undiluted (La Franca et al. 2002) and so dilution may not be the cause of all optically dull AGN. Another possibility is that the optical emission of elusive AGN has been rendered invisible by obscuration which may be caused be optically-thick gas clouds covering the nuclear source, as suggested by Comastri et al. (2002), or instead by extranuclear gas and dust in the host galaxy (Rigby et al. 2006). Alternatively, a fraction of elusive AGN are intrinsically optically weaker than other AGN (Trump et al. 2009) and can be characterised by unusual properties such as weak emission from the accretion disk (e.g. radiatively inefficient accretion flow RIAF, Yuan \& Narayan 2004). Finally more recent work by Castelló-Mor et al. (2012) proposes that the optical misclassification of X-ray bright AGN through emission lines may be due to the contamination of the narrow-line galaxy samples by narrow-line Seyfert 1 (NLS1), a subclass of unobscured AGN which have narrower line width (usually between 500 and $2000 \mathrm{~km} \mathrm{~s}^{-1}$ ) and a [OIII]/H $\beta$ line ratio smaller than 3 (Boller et al. 1996; Botte et al. 2004).

In this paper we investigate a new sample of Seyfert 2 (Sy2) galaxies with conflicting X-ray and optical classifications. After identifying possible NLS1, we consider three explanations for optical dullness of the remaining Sy2 galaxies in the sample: (1) obscuration by an dusty torus (Compton- thick AGN, $N_{\mathrm{H}}>$ $10^{24} \mathrm{~cm}^{-2}$ ); (2) optical dilution from the host galaxy star-light; (3) weak emission from the accretion disc related low accretion rates. 
Some of the AGN in our sample may belong to a more exotic class of AGN which are inconsistent with the predictions of the Unified Model (Antonucci 1993; Urry \& Padovani 1995). According to this scheme, AGN are classified into two classes based on the presence (type 1) or absence (type 2) of broad emission lines in their optical spectra. This means that the central black hole $(\mathrm{BH})$ is viewed directly (type 1) or is obscured by a dusty torus (type 2). However, type 2 AGN without an hidden broad line region (BLR) have been found (Bianchi et al. 2012); these are not predicted by the model. These sources, often called true Seyfert 2, may be observed while accreting at low Eddington rates, in agreement with models which predict that the BLR disappears below a certain accretion rate threshold (Yuan 2007) and the absence of observed broad-line AGN below a limiting accretion rate (corresponding to an Eddington ratio smaller than $10^{-2}$ ) (Trump et al. 2011).

The aim of this paper is to understand the nature of a new sample of AGN which have conflicting properties in the optical and X-ray band. This sample also allows us to find peculiar AGN with unexpected properties. The paper is structured as follows: Sect. 2 describes the source selection; the X-ray and optical properties are detailed in Sect. 3; and the Sect. 4 discusses the most likely explanations for optical dullness.

\section{Narrow emission-line galaxies sample}

\subsection{Source selection}

The 3XMM catalogue of serendipitous X-ray sources (Watson et al., in prep.) is currently the largest X-ray catalogue containing over 370000 discrete entries drawn from over 7000 pointed XMM-Newton observations covering around $2 \%$ of the sky. About $45 \%$ of $3 X M M$ catalogue sources lie in the region covered by the Sloan Digitial Sky Survey (SDSS). To create our sample of X-ray selected galaxies we carried out a positional cross-match of the 3XMM catalogue with SDSS-DR9, the ninth SDSS data release, focusing exclusively on galaxies with SDSS spectroscopy ( 1690000 objects). This allows us to create a large sample of sources with useful X-ray and optical data.

From SDSS we selected galaxies with a spectroscopic classification of GALAXY or QSO and which are science primary objects (i.e. those that have the best available spectra). The crossmatched X-ray/optical sample is obtained by cross-correlating the SDSS spectroscopic galaxies sub-sample with the 3XMM catalogue. SDSS galaxies are considered as potential counterparts to the X-ray sources if the separation between the optical and X-ray positions is smaller than $10^{\prime \prime}$ and for which the normalised separation (i.e. the ratio of the separation to the X-ray position error) is smaller than 4 (Pineau et al. 2011). Because we restrict our sample to SDSS spectroscopic objects, which are significantly brighter and thus have lower sky density than galaxies in the SDSS photometric catalogue, spurious matches become negligible even with these generous limits. In addition, X-ray sources are required to be point-like (3XMM catalogue parameter SC_EXTENT < 5; thus removing galaxy clusters) and to have a relatively high detection significance (catalogue parameter SC_DET_ML > 15). This results, at this stage, in a crossmatched catalogue of about 4900 X-ray objects with SDSS spectroscopic data.

From this sample, narrow emission-line galaxies are selected by requiring the full-width at half maximum (FWHM) of the Balmer lines to be smaller than $1000 \mathrm{~km} \mathrm{~s}^{-1}$ (e.g. Caccianiga et al. 2008); this threshold rejects all bona fide broad-line objects and reduces the contamination by conventional Narrow
Line Seyfert 1 (NLS1). Narrow emission-line galaxies represent $31 \%$ of the whole cross-matched catalogue, corresponding to 1555 sources.

Additional derived products for galaxies in the SDSS spectroscopic database are available from the MPA-JHU group. These are the GALPSPEC measurements (described in Brinchmann et al. 2004; Kauffmann et al. 2003b; Tremonti et al. 2004). The derived spectral products include emission line fluxes corrected for Galactic reddening (using the maps of Schlegel et al. 1998). In addition, through fitting of the observed spectrum with galaxy spectra models, the derived products provide estimates of emission line strengths after subtraction of stellar absorption line components. This is important because the SDSS aperture of the spectroscopic fibre is $3^{\prime \prime}$ and thus the spectra include not only the nucleus but have also host galaxy contribution. Moreover, using stellar population models, the derived products include a variety of galaxy parameters (e.g. stellar masses, stellar and gas kinematics, velocity dispersions ....). We choose to keep only sources for which these improved derived spectral products were available. Furthermore we removed from our sample those galaxies which are, SDSS X-ray targets (those selected from ROSAT, Chandra and XMM catalogues), together with objects from the SDSS BOSS sample (most of which, in our sample, are $\mathrm{X}$-ray selected or are selected because they other particular properties). Moreover sources which are the targets of XMM-Newton observations are also removed. We have made this selection because these SDSS and XMM targets would represent a biased subsample ${ }^{2}$. After the new selections have been applied the final sample consists of 1514 objects, corresponding to $97 \%$ of the narrow emission-line galaxies.

Finally, to have a trustworthy sample classification, galaxies are only considered if they have a reasonable quality optical spectrum (signal to noise ratio of the whole spectrum $S / N>3$ ) and a redshift $z<0.4$ (so that all of the four lines $\mathrm{H} \beta,[\mathrm{OIII}] \lambda 5007, \mathrm{H} \alpha$ and [NII] $\lambda 6584$ are covered). We further require that these lines are in emission with a reliable measurement; specifically the line ratios $[\mathrm{OIII}] / \mathrm{H} \beta$ and $[\mathrm{NII}] / \mathrm{H} \alpha$ must be three times larger than the error on the ratio.

Using these criteria, the final sample of X-ray selected narrow-emission line galaxies (NELGs) includes 797 X-ray sources with good SDSS spectroscopy and a median redshift of 0.15 .

\subsection{Classification}

\subsubsection{Optical classification}

The BPT diagnostic diagram is used to classify the NELGs sample on the basis of their optical emission properties. The classification scheme that best distinguishes between AGN and starforming galaxies uses the $[\mathrm{OIII}] / \mathrm{H} \beta$ vs. [NII] $/ \mathrm{H} \alpha$ emission-line ratios (Stasińska et al. 2006). The other main virtues of this scheme are that the lines used are relatively strong, lie in an easily accessible region of the optical spectrum and the line ratios are relatively insensitive to reddening because of their close separation in wavelength.

Two demarcation lines are used here for the classification. The first is the theoretical division from Kewley et al. (2001, hereafter Ke01), which uses a combination of photoionization and stellar population synthesis models to place a theoretical

\footnotetext{
2 An unbiased sample is required for future analysis using the same dataset.
} 
E. Pons and M. G. Watson: A new sample of X-ray selected narrow emission-line galaxies. I.
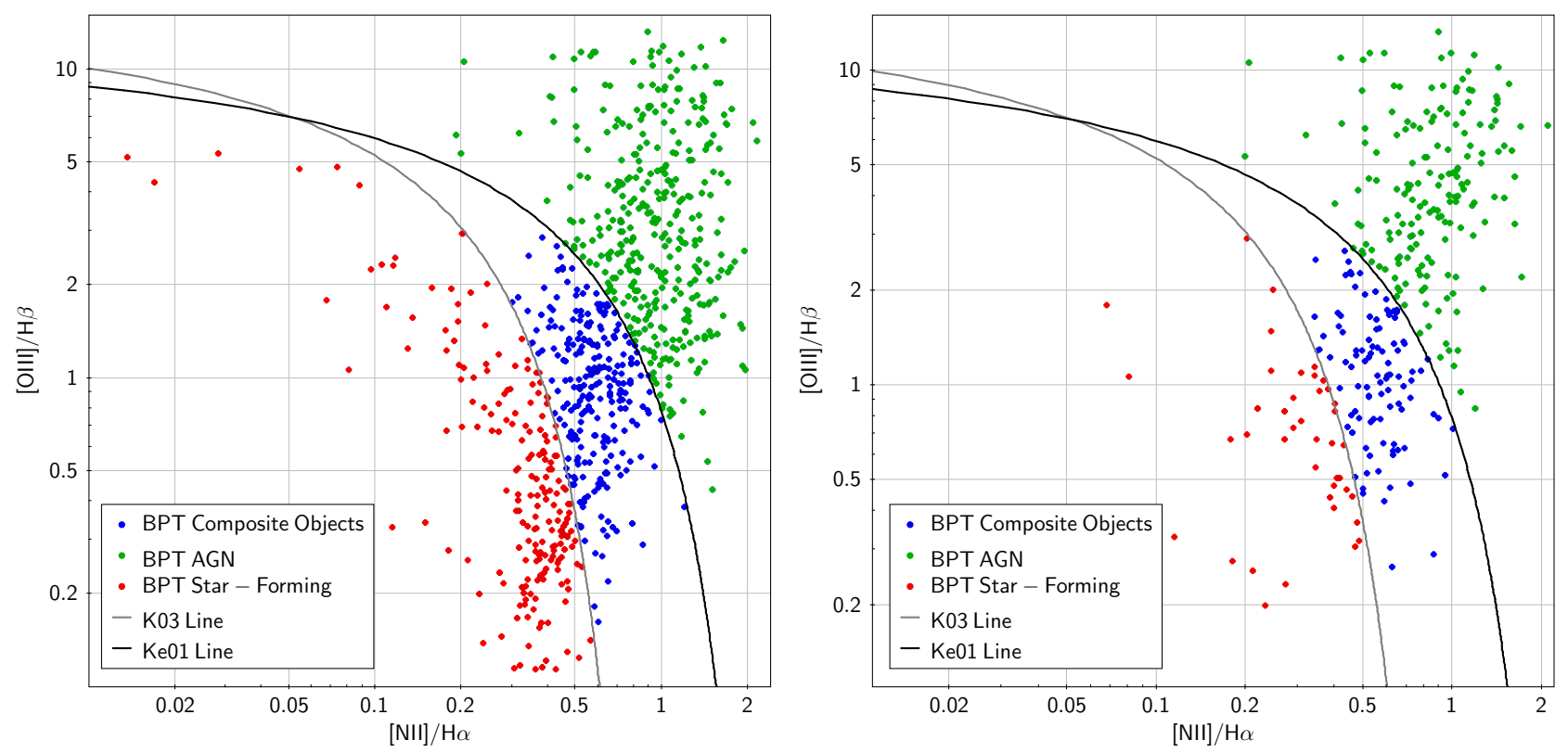

Fig. 1. BPT emission line diagnostic diagrams. The K03 line in grey separates the SF galaxies and composite object regions, while the Ke01 demarcation line in black distinguishes between optically-classified AGN and composite objects. Left panel: NELG sample which consists of 797 sources (45\% of BPT-AGN (green symbols), $31 \%$ composite objects (blue symbols) and $24 \%$ in the SF region (red symbols)). Right panel: NELG sample with high hard X-ray luminosity $\left(L_{\mathrm{HX}}>10^{42} \mathrm{ergs}^{-1}\right)$. There are 172 sources (54\% of the sample) classified as BPT-AGN (green symbols), 103 sources (blue symbols) classified as composite objects and 41 sources (13\%) in the SF region of the diagram (red symbols; our elusive AGN).

conservative lower limit on the true number of AGN:

$\log \left(\frac{[\mathrm{OIII}] \lambda 5007}{\mathrm{H} \beta}\right)=\frac{0.61}{\log \left(\frac{[\mathrm{NII}] \lambda 6584}{\mathrm{H} \alpha}\right)-0.47}+1.19$.

We also use an empirical line ratio based on the data from Kauffmann et al. (2003a, hereafter K03) that places a conservative limit on the true number of SF galaxies:

$\log \left(\frac{[\mathrm{OIII}] \lambda 5007}{\mathrm{H} \beta}\right)=\frac{0.61}{\log \left(\frac{[\mathrm{NII}] \lambda 6584}{\mathrm{H} \alpha}\right)-0.05}+1.3$.

The objects that lie below the K03 line are optically classified as SF galaxies (BPT-SF), the ones that are above the Ke01 line are classified as AGN (BPT-AGN). The objects that lie between these two lines are called Composite objects because their optical spectra may include a contributions from both starformation and an active nucleus. The classification for the whole NELG sample is shown in the left panel of the Fig. 1.

\subsubsection{X-ray classification}

An alternative way of classifying galaxies as AGN uses an empirical X-ray luminosity threshold. The rest-frame hard X-ray luminosity in the $2-10 \mathrm{keV}$ band $\left(L_{\mathrm{HX}}\right)$ is computed from the measured flux, making the simple assumption that the X-ray spectrum has a power-law form with a photon index $\Gamma$ of 1.7. The luminosity estimated this way is relatively insensitive to changes in the assumed photon index. Choosing sources with $L_{\mathrm{HX}}>10^{42} \mathrm{ergs}^{-1}$ is known to remove galaxies with X-ray emission powered by mechanisms other than nuclear activity (Fabbiano 1989; Mushotzky 2004). This empirical limit works because for a star-forming galaxy to have such a high X-ray luminosity requires a star formation rate (SFR) of at least $200 M_{\odot} \cdot \mathrm{yr}^{-1}$ (e.g. relation from Ranalli et al. 2003; $L_{\mathrm{HX}}=$ $\left.S F R\left[M_{\odot} \mathrm{yr}^{-1}\right] \cdot 5 \times 10^{39} \mathrm{erg} \mathrm{s}^{-1}\right)$. Such a high SFR corresponds to that of powerful Ultra Luminous Infrared Galaxies (ULIRGs; $S R F_{\text {min,ULIRG }} \sim 100 M_{\odot} \mathrm{yr}^{-1}$ ), objects which have a low space density, and thus are unlikely to be a significant contaminant at $z<0.4$. So selection with a threshold $L_{\mathrm{HX}}>10^{42} \mathrm{erg} \mathrm{s}^{-1}$ should produce a sample that only contains AGN. However, among the 316 NELGs with X-ray luminosity above this threshold, there remain 41 sources $(13 \%)$ that are optically classified as SF galaxies. We refer to these as elusive AGN (see Fig. 1, right panel).

The SFR provide in the SDSS GALSPEC catalogue (SFR SDSS $_{\text {). }}$

\section{X-ray vs. optical properties}

In this section we discuss the X-ray and optical properties of the elusive AGN.

\subsection{X-ray vs. [OIII] luminosity}

We compare the hard X-ray and [OIII] luminosities using [OIII] luminosities which are corrected for intrinsic absorption using the Balmer decrement, assuming an intrinsic ratio $(\mathrm{H} \alpha / \mathrm{H} \beta)_{0}$ of 3.1 in the NLR (see Osterbrock \& Ferland 2006) and a $\mathrm{H} \beta / \mathrm{H} \alpha$ colour index for extinction $\alpha=2.94$ (Bassani et al. 1999):

$f_{\text {corr }}=f_{\mathrm{obs}} \cdot\left(\frac{\mathrm{H} \alpha / \mathrm{H} \beta}{(\mathrm{H} \alpha / \mathrm{H} \beta)_{0}}\right)^{\alpha}$.

Compared to the BPT-AGN and composite objects in the sample, the elusive AGN have a mean thickness parameter $T$ ( $\left.T=L_{\mathrm{HX}} / L_{[\mathrm{OIII}]}\right)$ which is larger $\left(\log _{10} T \sim 2.1\right)$ than the other sources $\left(\log _{10} T \sim 1.5\right)$; but still in the typical range of values for Seyfert galaxies $\left(-1 \lesssim \log _{10} T \lesssim 2\right.$; Bassani et al. 1999) (see Fig. 2). This is consistent with the idea that some of the AGN in our sample could have anomalously low [OIII] flux (see also Trouille \& Barger 2010). 


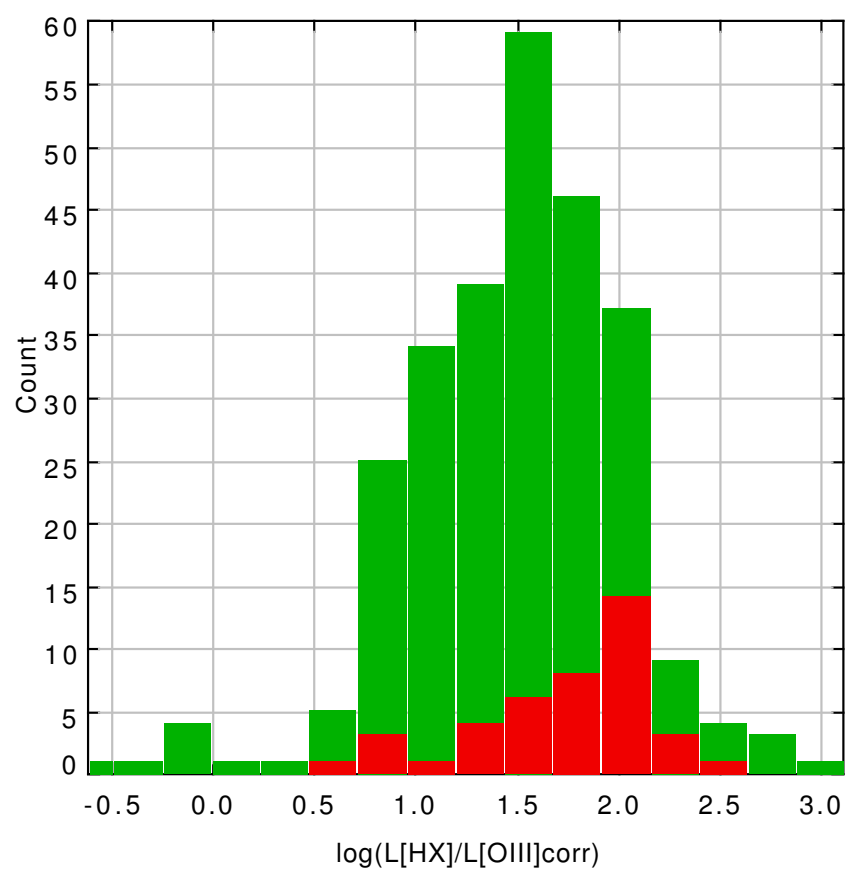

Fig. 2. $L_{\mathrm{H} X / L_{[\mathrm{O} I I], c o r r}}$ distribution for the BPT-AGN and composite objects (green) and the elusive AGN (red). The latter sources have a higher mean ratio compared to the other AGN in the sample.

\subsection{Emission line width vs. X-ray spectral information}

A crude indicator of the X-ray spectral properties can be deduced from the hardness ratio (HR) diagram (also called X-ray colourcolour diagram) which simply compares count rates in adjacent energy bands. The HR is defined in terms of the observed counts rates $R$ in energy bands $n$ and $n+1$ as follows:

$\mathrm{HR} n=\left(R_{n+1}-R_{n}\right) /\left(R_{n+1}+R_{n}\right) \quad$ for $n=1-4$

where the five energy bands are $0.2-0.5 \mathrm{keV}, 0.5-1.0 \mathrm{keV}$, 1.0-2.0 keV, 2.0-4.5 keV and 4.5-12 keV.

The HR3 vs. HR2 diagram, shown in Fig. 3, gives an approximate indicator of the intrinsic X-ray spectral shape and is also sensitive to the level of absorption. AGN with intrinsic absorption have a X-ray spectrum much harder than an unabsorbed one because the soft X-ray emission is differentially attenuated by the absorber. The dominant source of variation in the HR values is absorption (see Wang et al. 2004, Fig. 2). So an unabsorbed X-ray spectrum has HR2 and HR3 values lower than an absorbed one for a wide range of assumed continuum shapes. A typical type $1 \mathrm{AGN}$ has a HR2 value $\sim 0$ and HR3 $<0$; an increase in absorption above $10^{22} \mathrm{~cm}^{-2}$ would increase the HR values to HR2 0.4 and HR3 $~ 0$ (see Watson et al. 2009, Fig. 12). About $73 \%$ of the elusive AGN have low hardness ratios (HR2 $<0.4$ and HR3 $<0$ ), consistent with being unabsorbed or having low absorption.

The selected narrow-line AGN, even with a threshold of $1000 \mathrm{~km}^{-1}$ for the FWHM of the Balmer lines, could still be contaminated by NLS1. Indeed it has previously been noted that NLS1 can lie in the star-forming region of the BPT diagram (Osterbrock \& Pogge 1985; Rodríguez-Ardila et al. 2000) and that the lower end of their FWHM distribution can extend down to $500-600 \mathrm{~km} \mathrm{~s}^{-1}$ (Boller et al. 1996).

If we divide our elusive sample into broad line and narrow line sub-samples (hereafter referred as narrow and broad

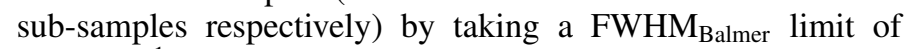
$600 \mathrm{~km} \mathrm{~s}^{-1}$, the 19 broader line sources $\left(\right.$ FWHM $_{\text {Balmer }}>$

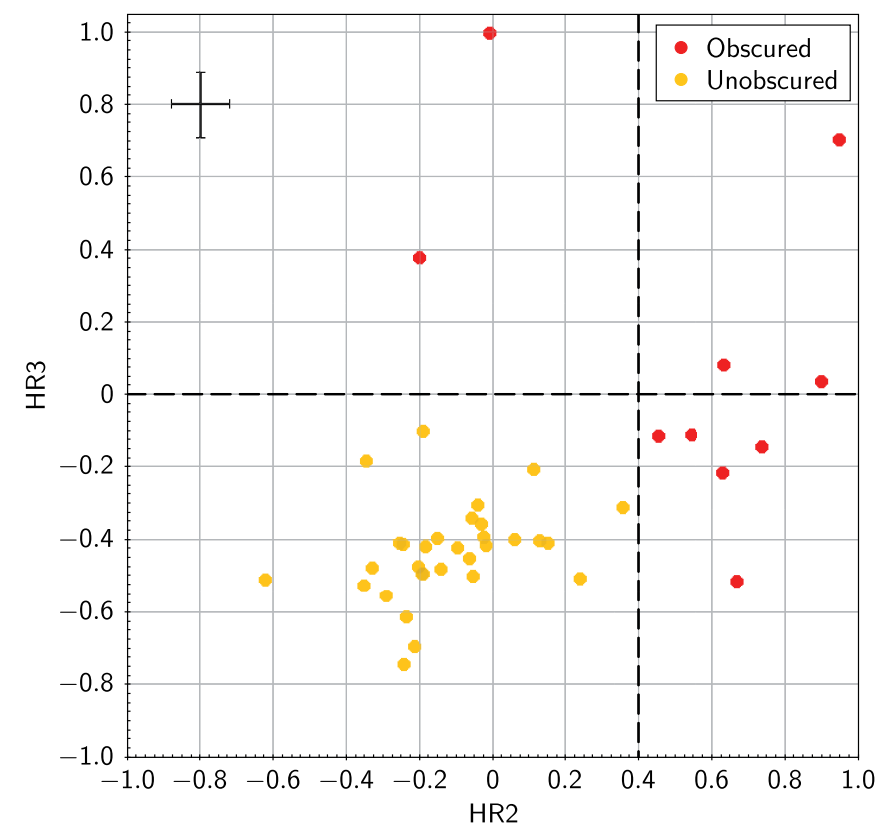

Fig. 3. X-ray colour-colour diagram (or HR diagram) of the elusive AGN. Gives a crude indication of the intrinsic X-ray spectral shape and level of absorption. The majority of the sources have a low HR (in yellow) consistent with being unabsorbed or having low absorption column density $N_{\mathrm{H}} \lesssim 10^{22} \mathrm{~cm}^{-2}$. The errors for both HR2 and HR3 range from about 0.01 to 0.2 , with a mean values of respectively 0.08 and 0.09 . The size of the typical (mean) errors is indicated by the black error-bars shown in the top left of the diagram.

$600 \mathrm{~km} \mathrm{~s}^{-1}$ ), all have a low HR (indicating low absorption) and so are candidates to be NLS1. On the other hand the nature of the sources with lower velocity widths is at this point unclear and only $41 \%$ of them show clear signs of absorption (i.e. have high HR; the others narrow sources with low HR are referred as the unobscured narrow sub-sample). Most of them also have a velocity width smaller than $350 \mathrm{~km} \mathrm{~s}^{-1}$ corresponding to characteristic values of host galaxy lines and so do not seem to be dominated by an AGN in the optical. Five sources have velocity width broader than expected for a host galaxy, so may be AGN dominated, but still below the broad limit chosen to distinguish between NLS1 and Sy2 $\left(F W H M_{\text {balmer }} \sim 400-600 \mathrm{~km} \mathrm{~s}^{-1}\right)$ (see Fig. 4). These five sources have an ambiguous classification based on their Balmer velocity line width only; they can be classical AGN dominated X-ray Sy2 or NLS1 but with very low line widths compared to common sources of this class. The separation between the broad and narrow sub-samples for these sources require further analysis and will be discussed in Sect. 3.4. At this stage possible NLS1 in the elusive sample are assumed to have line FWHM greater than $600 \mathrm{~km} \mathrm{~s}^{-1}$ and so belong to the broad sub-sample.

\subsection{X-ray spectral analysis}

An X-ray spectral analysis of the elusive AGN has been carried out based on the XMM-Newton EPIC data and using XSPEC (version 12.8.0). The source parameters and classification are given Table 1.

For the sources with more than 500 counts in the three EPIC cameras combined, spectra are already extracted as pipeline data products associated with the $3 \mathrm{XMM}$ catalogue. In this case, we use the EPIC-pn spectra from the PPS files with the corresponding latest version of the calibration files (SAS 11.0). 
E. Pons and M. G. Watson: A new sample of X-ray selected narrow emission-line galaxies. I.

Table 1. Observational parameters and classification of the elusive sources.

\begin{tabular}{|c|c|c|c|c|c|c|c|c|c|}
\hline $\begin{array}{l}3 \mathrm{XMM} \\
\text { SRCID }\end{array}$ & $\begin{array}{l}\text { IAU Name } \\
(3 \mathrm{XMM} \cdots)\end{array}$ & OBSID & $\begin{array}{r}t_{\text {exp }} \\
(\mathrm{ks})\end{array}$ & $\begin{array}{l}\text { EPIC-pn } \\
\text { counts }\end{array}$ & $\begin{array}{c}N_{\mathrm{H}, \mathrm{gal}} \\
\left(\times 10^{20} \mathrm{~cm}^{-2}\right)\end{array}$ & $\begin{array}{l}\text { Sub- } \\
\text { sample }^{(1)}\end{array}$ & $z^{(2)}$ & $\begin{array}{c}L_{\mathrm{HX}} \\
\left(\times 10^{42} \mathrm{erg} \mathrm{s}^{-1}\right)\end{array}$ & $\begin{array}{l}M_{r}^{(2)} \\
(\mathrm{mag})\end{array}$ \\
\hline 1780 & $\mathrm{~J} 121048.0+393745$ & 0112830201 & 58.9 & $331 \pm 14^{\diamond}$ & 2.13 & $\mathcal{N} 1(n \mathcal{N} 1)$ & 0.20 & $6.17 \pm 1.21$ & -22.3 \\
\hline 3161 & J094540.4+094749 & 0150970301 & 23.4 & $154 \pm 15^{\star}$ & 2.50 & $\mathcal{N}(n \mathcal{N})$ & 0.16 & $6.44 \pm 1.56$ & -22.2 \\
\hline 3173 & $\mathrm{~J} 103342.7+392926$ & 0506440101 & 87.8 & $498 \pm 26^{\circ}$ & 1.31 & $\mathcal{N}(n \mathcal{N})$ & 0.07 & $1.99 \pm 0.31$ & -21.5 \\
\hline 7207 & $\mathrm{~J} 095921.2+024030$ & 0302351601 & 30.5 & $2017 \pm 56$ & 1.76 & $\mathcal{B}(n \mathcal{B})$ & 0.26 & $16.97 \pm 1.45$ & -23.0 \\
\hline 18567 & J001804.8-005141 & 0403760701 & 27.1 & $1271 \pm 39$ & 2.99 & $\mathcal{B}(n \mathcal{B})$ & 0.19 & $7.27 \pm 1.29$ & -19.5 \\
\hline 18747 & $\mathrm{~J} 135810.3+653300$ & 0305920601 & 12.0 & $463 \pm 26$ & 1.38 & $\mathcal{B}(n \mathcal{B})$ & 0.23 & $8.84 \pm 1.65$ & -21.8 \\
\hline * 28357 & $\mathrm{~J} 134834.9+263109$ & 0097820101 & 34.3 & $42599 \pm 218$ & 1.21 & $\mathcal{B}(n \mathcal{B})$ & 0.06 & $8.24 \pm 0.16$ & -20.9 \\
\hline 28899 & J094240.9+480017 & 0201470101 & 11.9 & $393 \pm 22$ & 1.19 & $\mathcal{N} 1(n \mathcal{B})$ & 0.20 & $2.61 \pm 1.22$ & -21.1 \\
\hline 29911 & $\mathrm{~J} 100234.8+024253$ & 0203360101 & 26.0 & $416 \pm 25$ & 1.78 & $\mathcal{B}(n \mathcal{B})$ & 0.20 & $2.79 \pm 0.48$ & -21.4 \\
\hline 36405 & $\mathrm{~J} 231858.2+000621$ & 0305600601 & 16.0 & $169 \pm 15^{\star}$ & 3.90 & $\mathcal{B}(n \mathcal{B})$ & 0.32 & $12.84 \pm 7.03$ & -21.8 \\
\hline 47793 & $\mathrm{~J} 112758.2+583558$ & 0112810101 & 20.6 & $123 \pm 15^{\star}$ & 0.91 & $\mathcal{N} 1(n \mathcal{N} 1)$ & 0.18 & $1.32 \pm 0.53$ & -21.9 \\
\hline 88476 & J080455.5+241124 & 0203280201 & 6.3 & $311 \pm 19$ & 3.92 & $\mathcal{N} 1(n \mathcal{B})$ & 0.14 & $4.53 \pm 1.22$ & -22.0 \\
\hline 93560 & J015727.2-004041 & 0303110101 & 3.5 & $255 \pm 20^{\star}$ & 2.61 & $\mathcal{B}(n \mathcal{B})$ & 0.39 & $47.50 \pm 21.50$ & -22.3 \\
\hline 93640 & J015804.7-005222 & 0303110101 & 3.4 & $356 \pm 22^{\star}$ & 2.50 & $(n \mathcal{N} 1)$ & 0.08 & $1.18 \pm($ & -19.7 \\
\hline 115294 & J004335.9+001455 & 0303562201 & 4.4 & $155 \pm 14^{\star}$ & 1.72 & & 0.11 & $5.32 \pm 2.73$ & -21.4 \\
\hline 120992 & J002253.2+001659 & 040703 & 27.0 & $207 \pm 20^{\star}$ & 2.75 & $\mathcal{N})$ & 0.21 & $13.31 \pm 2.42$ & -21.4 \\
\hline 126294 & $.0-000839$ & 06053 & 7.3 & $596 \pm 26^{\diamond}$ & 3.35 & B) & 0.09 & $8.95 \pm 2.30$ & -20.8 \\
\hline 155684 & $0-001355$ & 0201120101 & 12.1 & $145 \pm 19^{\star}$ & 6.14 & N) & 0.22 & $1.71 \pm 0.71$ & -22.2 \\
\hline 155872 & J030705.8-000009 & 0142610101 & 40.3 & $1355 \pm 42$ & 6.27 & 3) & 0.27 & $10.20 \pm 1.62$ & -21.0 \\
\hline 266064 & $\mathrm{~J} 212929.6+000102$ & 0093030201 & 34.7 & $178 \pm 20^{\star}$ & 3.67 & $\mathcal{N}(n \mathcal{N})$ & 0.13 & $1.95 \pm 0.27$ & -21.4 \\
\hline 266125 & J213026.2-000204 & 0093030201 & 34.6 & $144 \pm 22^{\star}$ & 3.65 & $\mathcal{N} 1(n \mathcal{N} 1)$ & 0.14 & $1.77 \pm 0.89$ & -21.7 \\
\hline 275370 & $\mathrm{~J} 104549.6+213106$ & 0128531601 & 76.5 & $62 \pm 13^{\star}$ & 1.83 & $\mathcal{N} 1(n \mathcal{N} 1)$ & 0.14 & $1.17 \pm 0.62$ & -21.6 \\
\hline 277671 & J110738.9+520644 & 0304071201 & 5.9 & $167 \pm 16^{\star}$ & 0.94 & $\mathcal{N}(n \mathcal{N})$ & 0.18 & $17.49 \pm 4.78$ & -22.1 \\
\hline 282027 & $\mathrm{~J} 102812.6+293223$ & 0301650401 & 7.8 & $115 \pm 13^{\star}$ & 1.91 & $\mathcal{B}(n \mathcal{B})$ & 0.29 & $16.01 \pm 12.10$ & -21.7 \\
\hline 291676 & $\mathrm{~J} 115648.6+064753$ & 0301651801 & 4.0 & $305 \pm 19^{\star}$ & 1.14 & $\mathcal{N} 1(n \mathcal{B})$ & 0.15 & $3.18 \pm 2.85$ & -22.2 \\
\hline 294033 & $\mathrm{~J} 111443.6+525834$ & 0143650901 & 3.7 & $885 \pm 31$ & 0.97 & $\mathcal{B}(n \mathcal{B})$ & 0.08 & $3.60 \pm 0.86$ & -21.4 \\
\hline 297574 & $\mathrm{~J} 112405.1+061248$ & 0103863201 & 5.1 & $896 \pm 33$ & 4.61 & $\mathcal{B}(n \mathcal{B})$ & 0.27 & $28.25 \pm 4.80$ & -22.5 \\
\hline 303293 & J091440.9+435716 & 0604680201 & 14.3 & $148 \pm 18^{\star}$ & 1.45 & $\mathcal{N} 1(n \mathcal{N} 1)$ & 0.19 & $2.74 \pm 1.36$ & -22.3 \\
\hline 305003 & $\mathrm{~J} 084058.1+383300$ & 0502060201 & 16.1 & & 3.15 & $\mathcal{N} 1(n \mathcal{N} 1)$ & 0.12 & $19 \pm 0.61$ & -21.5 \\
\hline 321587 & J094057.1+032400 & 050201 & 22.3 & $213 \pm 76$ & 2.99 & $\mathcal{B}(n \mathcal{B})$ & 0.06 & $4.91 \pm 0.32$ & -20.8 \\
\hline 324308 & $\mathrm{~J} 13423$ & 010 & 25.6 & $33^{\circ}$ & 1.03 & $\mathcal{B}$ & 0.06 & $3.78 \pm 0.44$ & -20.9 \\
\hline 337999 & $\mathrm{~J} 151128.9+561317$ & 0670650401 & 7.4 & $655 \pm 29^{\star}$ & 1.38 & $\mathcal{N} 1(n \mathcal{B})$ & 0.15 & $16.48 \pm 2.91$ & -22.4 \\
\hline 339379 & $\mathrm{~J} 151525.0+042146$ & 0653810601 & 7.6 & $263 \pm 22^{\star}$ & 3.57 & $\mathcal{N} 1(n \mathcal{N} 1)$ & 0.10 & $3.91 \pm 0.54$ & -21.5 \\
\hline 341733 & $\mathrm{~J} 141449.5+361240$ & 0148620101 & 13.9 & $1217 \pm 17$ & 1.01 & $\mathcal{B}(n \mathcal{B})$ & 0.18 & $10.59 \pm 1.19$ & -21.4 \\
\hline 348033 & $\mathrm{~J} 123155.5+200333$ & 0301450201 & 24.1 & $67 \pm 11^{\star}$ & 2.58 & $\mathcal{N}(n \mathcal{N})$ & 0.11 & $1.51 \pm 0.74$ & -20.8 \\
\hline 350309 & $\mathrm{~J} 123748.5+092323$ & 0504100601 & 17.6 & $2224 \pm 51$ & 1.47 & $\mathcal{B}(n \mathcal{B})$ & 0.12 & $11.24 \pm 2.14$ & -21.7 \\
\hline 350410 & $\mathrm{~J} 123719.3+114915$ & 0112840101 & 14.5 & $247 \pm 20^{\star}$ & 2.83 & $\mathcal{N}(n \mathcal{N})$ & 0.11 & $2.26 \pm 0.34$ & -20.7 \\
\hline 354458 & J121613.0-032025 & 0305800701 & 38 & $136 \pm 14^{\star}$ & 2.66 & $\mathcal{N}(n \mathcal{N})$ & 0.13 & $2.36 \pm 0.73$ & -21.6 \\
\hline 356567 & $\mathrm{~J} 122349.5+072657$ & 0205090101 & 21.5 & $1288 \pm 39$ & 1.70 & $\mathcal{N} 1(n \mathcal{B})$ & 0.07 & $1.82 \pm 0.33$ & -21.6 \\
\hline 358675 & $\mathrm{~J} 121814.8+142601$ & 0147610101 & 13.4 & $456 \pm 24$ & 2.87 & $\mathcal{B}(n \mathcal{B})$ & 0.13 & $4.45 \pm 0.76$ & -20.1 \\
\hline 369702 & $\mathrm{~J} 125821.3+013158$ & 0658400601 & 14.0 & $2373 \pm 52$ & 1.45 & $\mathcal{B}(n \mathcal{B})$ & 0.16 & $2.51 \pm 0.64$ & -21.1 \\
\hline
\end{tabular}

Notes. ${ }^{(*)}$ This source is not an XMM-Newton target, nevertheless, detailed analysis of its serendipitous XMM data is presented by Zoghbi et al. (2008) (see also upper panel of Fig. 5). ${ }^{(1)}$ The sub-sample names $\mathcal{B}, \mathcal{N}$ and $\mathcal{N} 1$ correspond respectively to the broad, narrow and unobscured narrow sub-samples. The classification given in brackets is discussed later, in Sect. 3.4. (2) The errors on the redshift $z$ and the absolute $r$ magnitude $M_{r}$ are not shown because they are smaller than $1 \% .^{\left({ }^{\circ}\right)}$ EPIC-MOS counts; because there is no pn data for these sources. $\left.{ }^{\star}{ }^{\star}\right)$ Total EPIC counts.

For the other sources, the raw observation data files (ODF) were reduced and analysed using the Science Analysis System (SAS) tool (xmmselect version 2.65.2) using standard techniques. The spectral data were extracted from an optimised circular region, and the corresponding background files from a nearby circular region free of sources. Because of the low number of counts, to improve the analysis, the EPIC-pn and EPICMOS spectra are analysed simultaneously with a constant coefficient inserted to model calibration uncertainties between the detectors (coefficient fixed to 1 for the pn and allowed to vary for the MOS data). In the few cases for which pn spectra are not available, we use the MOS data.

The spectral data are grouped with a minimum of 20 counts per bin in order to use the $\chi^{2}$ fit statistics. However, for the lowest quality spectra, data are grouped with only one count per bin and, in this case, we use Cash statistics (Cash 1979).
The classical model of the X-ray spectrum of an AGN is a power-law modified by absorption, with in some cases a soft X-ray excess (usually present in NLS1 spectra) and a Fe $\mathrm{K} \alpha$ emission line around $6.4 \mathrm{keV}$. The absorbed power-law comes from the Comptonization of photons from the accretion disc by a corona of hot material. The soft X-ray excess may be attributed to the blurred ionized reflection from the inner parts of the accretion disc (Gierliński \& Done 2004; Crummy et al. 2006) or to the Comptonization of extreme UV accretion disc photons (Ross et al. 1992). The Fe K $\alpha$ fluorescent emission-line is a signature of X-ray reprocessing (reflection of the power-law component by a cold disc; accretion disc or torus).

The spectral fitting is performed as follows:

1. We first fit the data in the $2-10 \mathrm{keV}$ energy range with a power-law (zpo model in XSPEC syntax) corrected for foreground absorption in our own Galaxy (tbabs; $N_{\mathrm{H}, \mathrm{gal}}$ ) and 


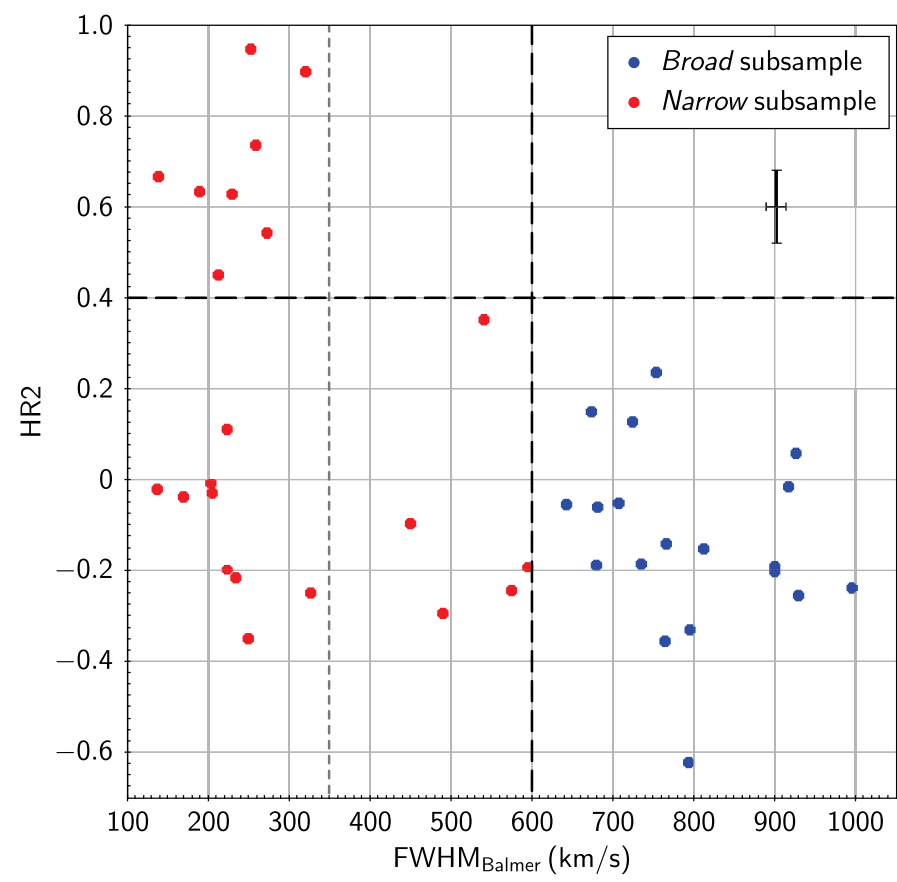

Fig. 4. HR2 vs. Balmer velocity width of elusive AGN. A total of 19 sources (blue symbols) do not show clear signs of absorption and have broader line widths; they are NLS1 candidates (broad sub-sample). The remaining 22 sources with narrower line widths (red symbols; narrow sub-sample) show different properties, only $41 \%$ of them exhibit clear signs of absorption. The size of the typical (mean) errors is indicated by the black error-bars shown in the top right of the diagram.

modified by intrinsic absorption (pha; $N_{\mathrm{H}}$ ). The $N_{\mathrm{H}, \mathrm{gal}}$ parameter is fixed to the value computed using the emphnh ftool (see Table 1), while $N_{\mathrm{H}}$ is free to vary.

2. We then check for the presence of an additional Fe emission line around $6.4 \mathrm{keV}$, which can be modelled by a component with a Gaussian profile (zgauss).

3. We finally include the data between 0.3 and $2 \mathrm{keV}$ to see if these data are in excess compared to the previously fitted power-law. If this is the case, we add a blackbody component (zbbody) to model the soft excess.

The best fit model is the one that gives the smallest reduced$\chi^{2}\left(=\chi^{2} /\right.$ d.o.f $)$. When the best fit model is the combination of 2 components (i.e. an absorbed power-law plus a soft excess and/or a Fe line), we use the XSPEC Ftest task to determine whether the inclusion of an additional component is needed. We also check the validity of the model using the XSPEC goodness parameter which returns the percentage of simulations for which the statistic is less than that for the observed data; so a smaller goodness parameter values indicates a better model (but this can only be used for $\chi^{2}$ statistic). We choose to keep the additional component if the F-test probability $P$ (ftest) $<10^{-2}$, or if the goodness parameter is really improved (i.e. a parameter difference of at least $20 \%$ ).

The results of the spectral fitting are given Table 2 . In the case of very low quality spectra, the number of counts was not sufficient to check the presence of a soft excess. Two example spectra (one for a source from the emphbroad sub-sample and one from the narrow sub-sample) are shown in Fig. 5.

A Seyfert galaxy is expected to have a photon index of about 1.8 (Nandra et al. 1997), while NLS1 have a softer X-ray slope, steeper than that of classical Seyferts (Botte et al. 2004). The
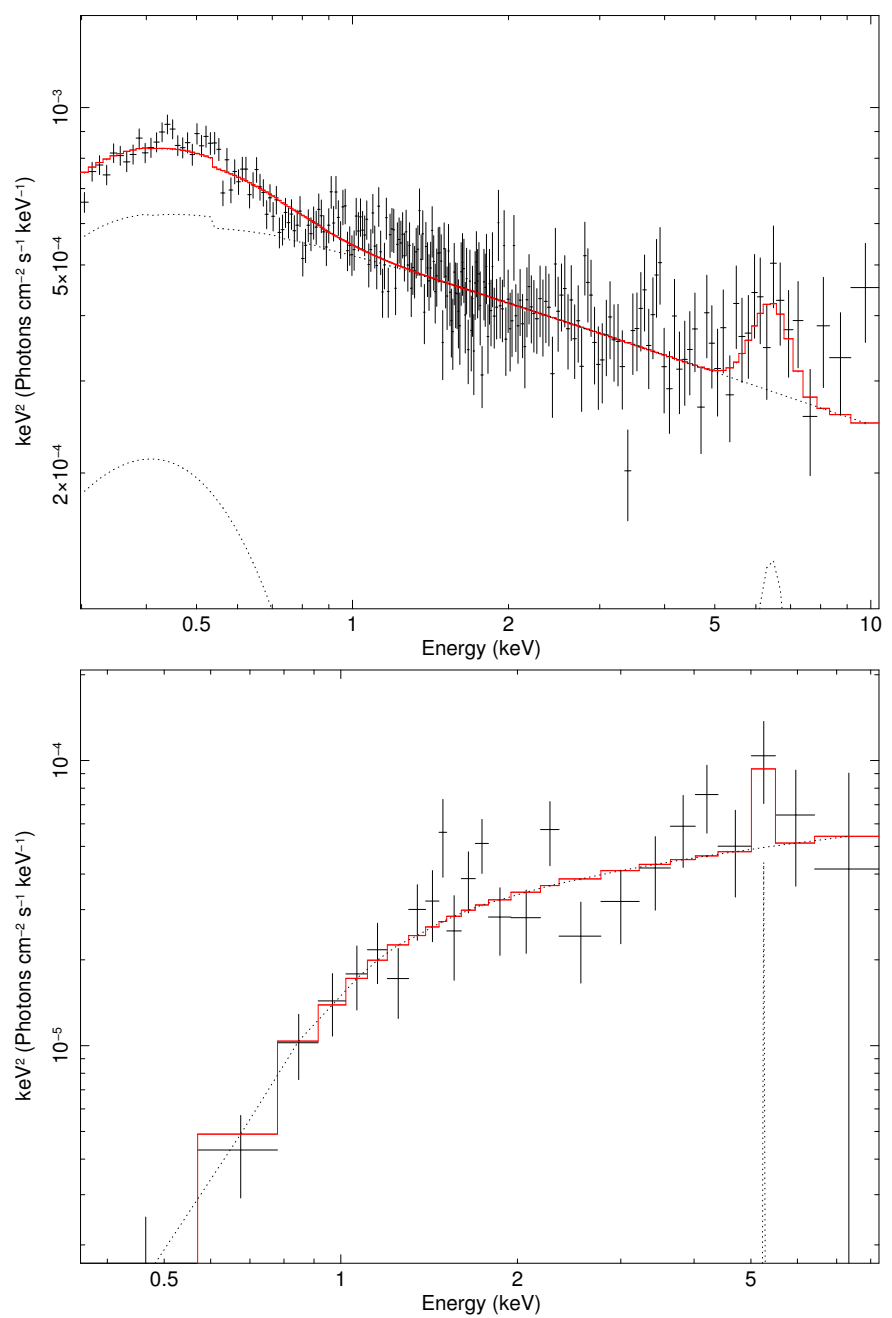

Fig. 5. Example X-ray spectra. Upper panel: NLS1 candidate (SRCID 28357) fitted with a power-law plus a blackbody component for the soft excess and a Gaussian component for the Fe line. Lower panel: a narrow source (SRCID 3173) fitted with a Gaussian component (Fe line) in addition to an absorbed power law. The dashed lines represent the separate model components while the red line is the resulting best fit model.

sources from the broad sub-sample (i.e. NLS1 candidates) have a photon index $\Gamma$ consistent with what is expected for $\operatorname{NLS} 1(\Gamma>$ $1.8)$ and have no or little intrinsic absorption $\left(N_{\mathrm{H}}<4 \times 10^{21} \mathrm{~cm}^{-2}\right.$ is often taken to be the separation between absorbed and unabsorbed AGNs; Caccianiga et al. 2007); strongly supporting their classification. The sources from the narrow sub-sample, with high HR2 $(>0.4)$, have $\Gamma<1.8$ and show signs of absorption in their spectra, so their X-ray emission resemble that of classical Seyfert 2 (Sy2) galaxies; while the others (unobscured narrow sub-sample) have a $\Gamma \gtrsim 1.8$ and their X-ray spectra alone cannot distinguish between NLS1 and Sy2 (see Fig. 6). The X-ray spectral analysis nevertheless confirms that the sample sources with a low HR do not show signs of absorption or have little intrinsic absorption.

\subsection{Forbidden vs. permitted lines width}

To better distinguish between Sy2 and NLS1 (especially for the sources from the unobscured narrow group with $\Gamma \sim 1.8$ ), we examine one of the NLS1 properties, the emission line width. 
E. Pons and M. G. Watson: A new sample of X-ray selected narrow emission-line galaxies. I.

Table 2. X-ray spectral analysis results.

\begin{tabular}{|c|c|c|c|c|c|c|c|c|c|}
\hline SRCID & Model & $\Gamma$ & $k T(\mathrm{keV})$ & $N_{\mathrm{H}}\left(\times 10^{22} \mathrm{~cm}^{-2}\right)^{(1)}$ & Red- $\chi^{2}$ & Goodness & $P($ ftest $)$ & $\mathrm{FeK}$ & Sub-sample ${ }^{(2)}$ \\
\hline 7207 & (2) & $2.40_{-0.41}^{+0.45}$ & $0.12_{-0.04}^{+0.08}$ & $0.12_{-0.11}^{+0.21}$ & 1.218 & $31 \%$ & 0.019 & $\checkmark$ & $\mathcal{B}(n \mathcal{B})$ \\
\hline 18567 & (2) & $2.19_{-0.26}^{+0.27}$ & $0.10_{-0.02}^{+0.02}$ & $<0.01$ & 1.176 & $44 \%$ & 0.004 & & $\mathcal{B}(n \mathcal{B})$ \\
\hline 18747 & (1) & $2.21_{-0.22}^{+0.20}$ & - & $<0.01$ & 0.608 & $8 \%$ & - & & $\mathcal{B}(n \mathcal{B})$ \\
\hline 28357 & (2) & $2.38_{-0.05}^{-0.22}$ & $0.10_{-0.01}^{+0.01}$ & $<0.01$ & 1.013 & $15 \%$ & 0.006 & $\checkmark$ & $\mathcal{B}(n \mathcal{B})$ \\
\hline 29911 & (2) & $1.67_{-0.79}^{-0.05}$ & $0.22_{-0.06}^{+0.01}$ & $<0.01$ & 0.853 & $15 \%$ & 0.298 & $\checkmark$ & $\mathcal{B}(n \mathcal{B})$ \\
\hline $93560^{*}$ & (1) & $2.51_{-0.54}^{+0.64}$ & - & $0.25_{-0.16}^{+0.20}$ & $1.283^{\bullet}$ & - & - & & $\mathcal{B}(n \mathcal{B})$ \\
\hline $115294^{*}$ & (1) & $2.17_{-0.30}^{+0.34}$ & - & $<0.01$ & $0.840^{\bullet}$ & - & - & & $\mathcal{B}(n \mathcal{B})$ \\
\hline 126294 & (2) & $2.30_{-0.28}^{+0.30}$ & $0.038_{-0.033}^{+0.0037}$ & $<0.01$ & 1.14 & $26 \%$ & 0.212 & & $\mathcal{B}(n \mathcal{B})$ \\
\hline 155872 & (2) & $2.03_{-0.43}^{+0.48}$ & $0.15_{-0.04}^{+0.02}$ & $<0.01$ & 1.106 & $35 \%$ & 0.004 & $\checkmark$ & $\mathcal{B}(n \mathcal{B})$ \\
\hline $282027^{*}$ & (1) & $2.40_{-0.46}^{+0.49}$ & - & $<0.01$ & $1.332^{\bullet}$ & - & - & & $\mathcal{B}(n \mathcal{B})$ \\
\hline 294033 & (2) & $2.03_{-0.53}^{+0.56}$ & $0.13_{-0.02}^{+0.02}$ & $<0.01$ & 0.914 & $15 \%$ & 0.052 & & $\mathcal{B}(n \mathcal{B})$ \\
\hline 297574 & (2) & $2.03_{-0.35}^{+0.36}$ & $0.13_{-0.03}^{+0.02}$ & $<0.01$ & 0.848 & $13 \%$ & 0.0004 & $\checkmark$ & $\mathcal{B}(n \mathcal{B})$ \\
\hline 321587 & (2) & $2.21_{-0.13}^{+0.33}$ & $0.12_{-0.02}^{+0.02}$ & $<0.01$ & 1.033 & $24 \%$ & 0.014 & $\checkmark$ & $\mathcal{B}(n \mathcal{B})$ \\
\hline 324308 & (1) & $1.98_{-0.10}^{+0.13}$ & - & $<0.01$ & 1.064 & $34 \%$ & - & & $\mathcal{B}(n \mathcal{B})$ \\
\hline 341733 & (2) & $1.88_{-0.22}^{+0.10}$ & $0.14_{-0.06}^{+0.03}$ & $<0.01$ & 0.905 & $14 \%$ & 0.051 & & $\mathcal{B}(n \mathcal{B})$ \\
\hline 350309 & (2) & $2.19_{-0.22}^{+0.22}$ & $0.09_{-0.02}^{+0.00}$ & $<0.01$ & 1.008 & $24 \%$ & 0.0003 & & $\mathcal{B}(n \mathcal{B})$ \\
\hline 358675 & (2) & $1.66_{-0.20}^{+0.20}$ & $0.04_{-0.02}^{+0.02}$ & $<0.01$ & 1.395 & $53 \%$ & 0.029 & & $\mathcal{B}(n \mathcal{B})$ \\
\hline $36405^{*}$ & (1) & $2.29_{-0.26}^{+0.20}$ & - & $<0.01$ & $0.770^{\bullet}$ & - & - & & $\mathcal{B}(n \mathcal{B})$ \\
\hline 369702 & (2) & $2.19_{-0.42}^{+0.46}$ & $0.12_{-0.06}^{+0.05}$ & $<0.01$ & 1.033 & $18 \%$ & $1.6 \cdot 10^{-6}$ & & $\mathcal{B}(n \mathcal{B})$ \\
\hline $3161^{*}$ & (1) & $0.92_{-0.21}^{-0.42}$ & - & $<0.14$ & $0.912^{\bullet}$ & - & - & & $\mathcal{N}(n \mathcal{N})$ \\
\hline 3173 & (1) & $1.74_{-0.37}^{+0.43}$ & - & $0.41_{-0.18}^{+0.24}$ & 1.056 & $25 \%$ & - & $\checkmark$ & $\mathcal{N}(n \mathcal{N})$ \\
\hline $120992^{*}$ & (1) & $1.99_{-1.31}^{+1.31}$ & - & $7.11_{-4.42}^{+7.28}$ & $0.663^{\bullet}$ & - & - & $\checkmark$ & $\mathcal{N}(n \mathcal{N})$ \\
\hline $155684^{*}$ & (1) & $1.54_{-0.63}^{+1.18}$ & - & $<1.81$ & $1.119^{\circ}$ & - & - & & $\mathcal{N}(n \mathcal{N})$ \\
\hline $266064^{*}$ & (1) & $1.22_{-1.17}^{+1.51}$ & - & $23.3_{-13.8}^{+22.8}$ & $0.971^{\bullet}$ & - & - & & $\mathcal{N}(n \mathcal{N})$ \\
\hline $277671^{*}$ & (1) & $0.79_{-0.25}^{+0.25}$ & - & $<0.11$ & $0.709^{\bullet}$ & - & - & & $\mathcal{N}(n \mathcal{N})$ \\
\hline $348033^{*}$ & (1) & $1.62_{-0.70}^{+1.64}$ & - & $<1.61$ & $0.701^{\bullet}$ & - & - & & $\mathcal{N}(n \mathcal{N})$ \\
\hline $350410^{*}$ & (1) & $0.97_{-0.42}^{+0.57}$ & - & $0.42_{-0.33}^{+0.69}$ & $0.723^{\bullet}$ & - & - & $\checkmark$ & $\mathcal{N}(n \mathcal{N})$ \\
\hline $354458^{*}$ & (1) & $1.87_{-0.39}^{+0.43}$ & - & $<0.04$ & $1.008^{\bullet}$ & - & - & & $\mathcal{N}(n \mathcal{N})$ \\
\hline 1780 & (1) & $2.47_{-0.45}^{+0.51}$ & - & $<0.11$ & 0.914 & $33 \%$ & - & & $\mathcal{N} 1(n \mathcal{N} 1)$ \\
\hline 28899 & (1) & $2.92_{-0.23}^{+0.45}$ & - & $<0.01$ & 0.536 & $4 \%$ & - & & $\mathcal{N} 1(n \mathcal{B})$ \\
\hline $47793^{*}$ & (1) & $1.96_{-0.36}^{+0.43}$ & - & $<0.08$ & $0.669^{\bullet}$ & - & - & & $\mathcal{N} 1(n \mathcal{N} 1)$ \\
\hline 88476 & (2) & $1.94_{-0.30}^{+0.30}$ & $0.07_{-0.03}^{+0.04}$ & $<0.01$ & 0.508 & $1 \%$ & 0.013 & & $\mathcal{N} 1(n \mathcal{B})$ \\
\hline 93640 & (2) & $2.24_{-0.21}^{+0.20}$ & $0.016_{-0.006}^{+0.078}$ & $<0.05$ & $0.764^{\bullet}$ & - & 0.012 & & $\mathcal{N} 1(n \mathcal{N} 1)$ \\
\hline $266125^{*}$ & (1) & $2.07_{-0.47}^{+0.52}$ & - & $<0.26$ & $1.006^{\bullet}$ & - & - & & $\mathcal{N} 1(n \mathcal{N} 1)$ \\
\hline $275370^{*}$ & (1) & $6.90_{-4.16}^{+}$ & - & $<0.01$ & $0.577^{\bullet}$ & - & - & & $\mathcal{N} 1(n \mathcal{N} 1)$ \\
\hline $291676^{*}$ & (1) & $3.02_{-0.36}^{+0.63}$ & - & $<0.09$ & $0.958^{\bullet}$ & - & - & & $\mathcal{N} 1(n \mathcal{B})$ \\
\hline $303293^{*}$ & (1) & $1.71_{-0.38}^{+0.46}$ & - & $<0.01$ & $1.029^{\circ}$ & - & - & & $\mathcal{N} 1(n \mathcal{N} 1)$ \\
\hline 305003 & (1) & $2.33_{-0.11}^{+0.12}$ & - & $<0.03$ & 0.839 & $4 \%$ & - & & $\mathcal{N} 1(n \mathcal{N} 1)$ \\
\hline 337999 & (1) & $1.83_{-0.25}^{+0.28}$ & - & $0.19_{-0.09}^{+0.11}$ & 1.241 & $47 \%$ & - & & $\mathcal{N} 1(n \mathcal{B})$ \\
\hline $339379^{*}$ & (1) & $1.07_{-0.38}^{+0.40}$ & - & $<0.01$ & $1.089^{\bullet}$ & - & - & & $\mathcal{N} 1(n \mathcal{N} 1)$ \\
\hline 356567 & (1) & $2.66_{-0.12}^{+0.13}$ & - & $<0.01$ & 0.826 & $13 \%$ & - & & $\mathcal{N} 1(n \mathcal{B})$ \\
\hline
\end{tabular}

Notes. All quoted errors are for a $90 \%$ confidence interval for one parameter. ${ }^{(1)}$ The lower limit of 0.1 on $N_{\mathrm{H}}$ corresponds to the limit imposed by XSPEC. ${ }^{(2)}$ The sub-sample names $\mathcal{B}, \mathcal{N}$ and $\mathcal{N} 1$ correspond respectively to the broad, narrow and unobscured narrow sub-samples. The classification given in brackets is discussed later, in Sect. 3.4. ${ }^{(*)}$ Very low number of counts (Cash statistic is used instead of $\chi^{2}$ statistic), not sufficient to check the presence of a soft excess. ${ }^{(\bullet)}$ Test statistic value when the Cash fit statistic is used. Model (1) corresponds to an absorbed power-law (tbabs*(zpo*pha)) and model (2) to a soft excess in addition to an absorbed power-law (tbabs*( (zpo+zbb)*pha)).

Because in NLS1 the broad lines come from the BLR, which is presumed to be closer to the BH than the NLR, they have permitted line widths larger that for the forbidden lines (Botte et al. 2004). As we can see in Fig. 7 there are clearly two kinds of sources. Some have the same line width for the permitted and forbidden lines (these we call the X-ray Seyfert 2 class; 41\%) whilst others have larger permitted line widths compared to their forbidden lines (NLS1 class; 59\%). The NLS1 class, (referring to $n \mathcal{B}$ in Tables 1 and 2), consists of all the sources from the broad sub-sample (i.e. NLS1 candidates) plus the five sources of the unobscured narrow sub-sample which have Balmer velocity line width not dominated by the host galaxy. The X-ray Sy2 sources (named as $n \mathcal{N}$ in Tables 1 and 2) are the new narrow sources, with 8 of them having a low $\mathrm{HR}(n \mathcal{N} 1$ classification in Tables 1 and 2, hereafter called unobscured X-ray Sy2) and so seem to be unabsorbed. 


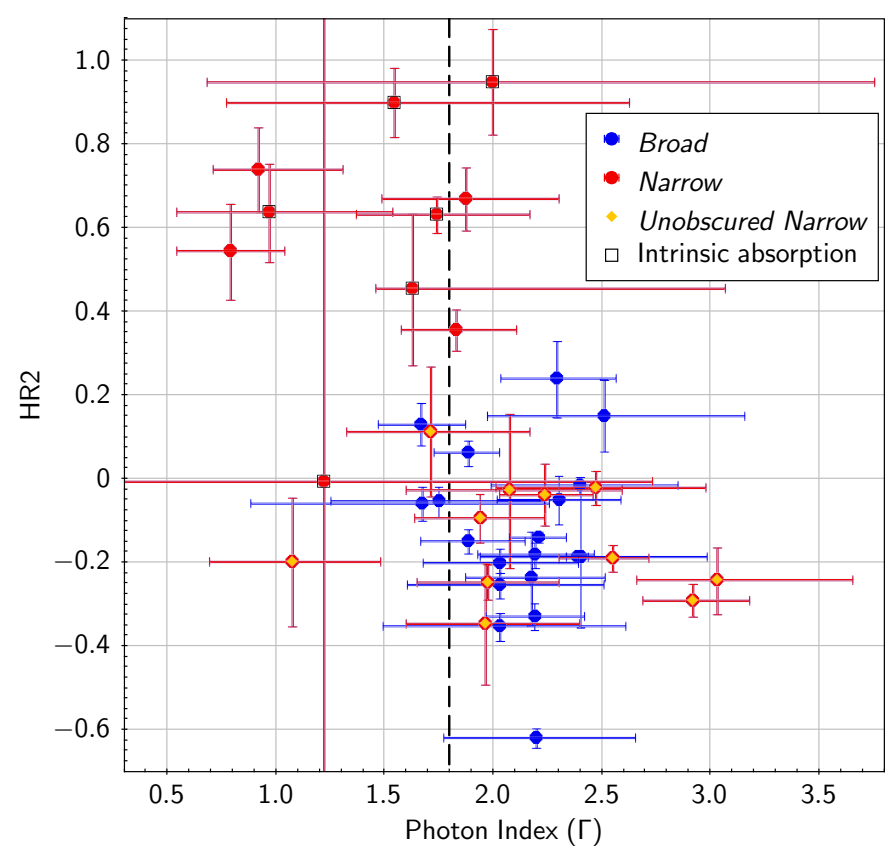

Fig. 6. HR2 vs. X-ray photon index. NLS1 candidates (in blue) have $\Gamma$ mostly larger than 1.8 and are unabsorbed, as expected. For narrow-line sources (in red), some have typical properties of Seyfert $2(\Gamma<1.8$ and intrinsic absorption) while the others, with low HR2, cannot be clearly classified using their photon index. One of the sources with very large photon index value and error is not plotted.

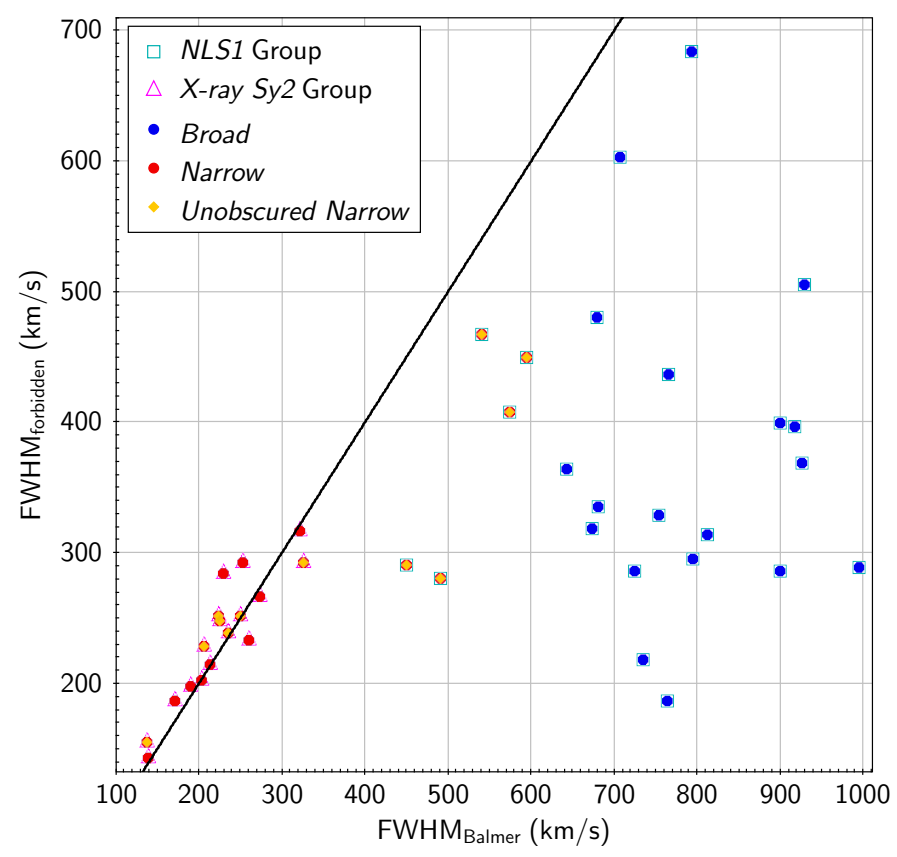

Fig. 7. Forbidden vs. permitted line widths. The black line corresponds to equal width. This comparison is used to distinguish between X-ray Sy2 (sources with equal line width) and NLS1 (which consists of all the broad sources (blue symbols) plus 5 sources from the unobscured narrow group).

\subsection{NLS1 class: black hole mass and Eddington ratio}

To confirm the classification of the NLS1 sources, we compute their BH masses and Eddington ratios $\left(\lambda=L_{\mathrm{bol}} / L_{\mathrm{Edd}}\right)$. NLS1 are known to be AGN with very low mass BH and must accrete at
near-Eddington rates $(\lambda \gtrsim 0.25$; Netzer \& Trakhtenbrot 2007) in order to maintain an AGN-like luminosity.

NLS1 share the same stellar velocity dispersion range as type 1 AGN in the $M_{\mathrm{BH}}-\sigma$ relation (Botte et al. 2004), so we can use the same BH mass relation as for unobscured AGN to estimate the mass of their central object. The mass of a $\mathrm{BH}$ can be computed assuming virial equilibrium: $M_{\mathrm{BH}}=R_{\mathrm{BLR}} v^{2} G^{-1}$. We use the scaling relation of Xiao et al. (2011):

$M_{\mathrm{BH}}\left(\mathrm{M}_{\odot}\right)=3.47 \cdot\left[\frac{\lambda L_{5100 \AA}}{10^{44} \mathrm{erg} \mathrm{s}^{-1}}\right]^{0.519} \cdot\left[\frac{F W H M_{\mathrm{H} \alpha}}{\mathrm{km} \mathrm{s}^{-1}}\right]^{2.06}$.

In this relationship, the size of the BLR, $\left(R_{\mathrm{BLR}}\right)$, is inferred from the AGN continuum luminosity at $5100 \AA$ using the revised relation from Bentz et al. (2009) calibrated using $\mathrm{H} \beta$. Then $\mathrm{FWHM}_{\mathrm{H} \beta}$ is converted to $\mathrm{FWHM}_{\mathrm{H} \alpha}$ according to the empirical relationship derived in Greene \& Ho (2005). Finally Xiao et al. (2011) assume an isotropic distribution of orbits with random inclinations $(v=f \cdot F W H M=\sqrt{3} / 2 \cdot F W H M)$. The luminosity $\lambda L_{5100}$ is the rest-frame luminosity corrected for Galactic absorption and host galaxy stellar contribution. The FWHM measurement for my sample comes from the SDSS GALSPEC spectral products and corresponds to the value fitted simultaneously for all of the Balmer lines.

The Eddington ratio only depends on the Eddington luminosity $L_{\text {Edd }}$ and on the bolometric luminosity $L_{\text {bol }}$. The Eddington luminosity is directly linked to the $\mathrm{BH}$ mass:

$L_{\text {Edd }}=1.25 \times 10^{38}\left(\frac{M_{\mathrm{BH}}}{M_{\odot}}\right) \mathrm{erg} \mathrm{s}^{-1}$.

Because our sample have low [OIII] luminosities for AGN and the mid-infrared emission can be contaminated by SF, the best choice to compute the bolometric luminosity is to use the hard X-ray luminosity $\left(L_{\mathrm{bol}}=L_{\mathrm{HX}} \cdot C_{\mathrm{HX}}\right)$. The conversion factor $C_{\mathrm{HX}}$ depends on $L_{\mathrm{HX}}$ (Vasudevan \& Fabian 2007) and can be estimated using the relation from Marconi et al. (2004):

$\log \left(\frac{L_{\mathrm{bol}}}{L_{\mathrm{HX}}}\right)=1.54+0.24 \mathcal{L}+0.012 \mathcal{L}^{2}-0.0015 \mathcal{L}^{3}$

where $\mathcal{L}=\log \left(L_{b o l}\right)-12$, and $L_{b o l}, L_{H X}$ are in units of $L_{\odot}$.

Using Eqs. (1)-(3), the sources from the NLS1 group have low mass BH $\left(M_{\mathrm{BH}}<5 \times 10^{6} M_{\odot}\right)$; compared to a typical AGN which has $M_{\mathrm{BH}} \sim 10^{7}-10^{9} M_{\odot}$; and also have a high Eddington ratio $(\lambda \gtrsim 0.25)$ as expected, as shown in Fig. 8.

Among the elusive AGN, half of sources (59\%) are likely to be NLS1 and are optically misclassified as star-forming galaxies due to the fact that Balmer lines $(\mathrm{H} \alpha$ and $\mathrm{H} \beta$ ) come from both the NLR and BLR regions and therefore cannot be correctly classified using the BPT diagram. However, we still have $41 \%$ of the sample that do not appear to be NLS1, and mostly resemble $\mathrm{X}$-ray type $2 \mathrm{AGN}$, in the sense that they do not possess optical broad lines and have AGN X-ray luminosity (in contrast with the work of Castelló-Mor et al. 2012, who found that the population of elusive AGN is entirely comprised of NLS1). Possible reasons of their optical misclassification are discussed in the next section.

\section{Why type 2 AGN could be optically misclassified as SF galaxies}

We consider three possible explanations for the fact that the X-ray Sy2 galaxies (i.e. the 17 sources with $F W H M_{\text {Balmer }}<$ $400 \mathrm{~km} \mathrm{~s}^{-1}$ ) are not apparent as AGN in the optical band: obscuration, optical dilution by host galaxy starlight and weak emission from the AGN. 
E. Pons and M. G. Watson: A new sample of X-ray selected narrow emission-line galaxies. I.

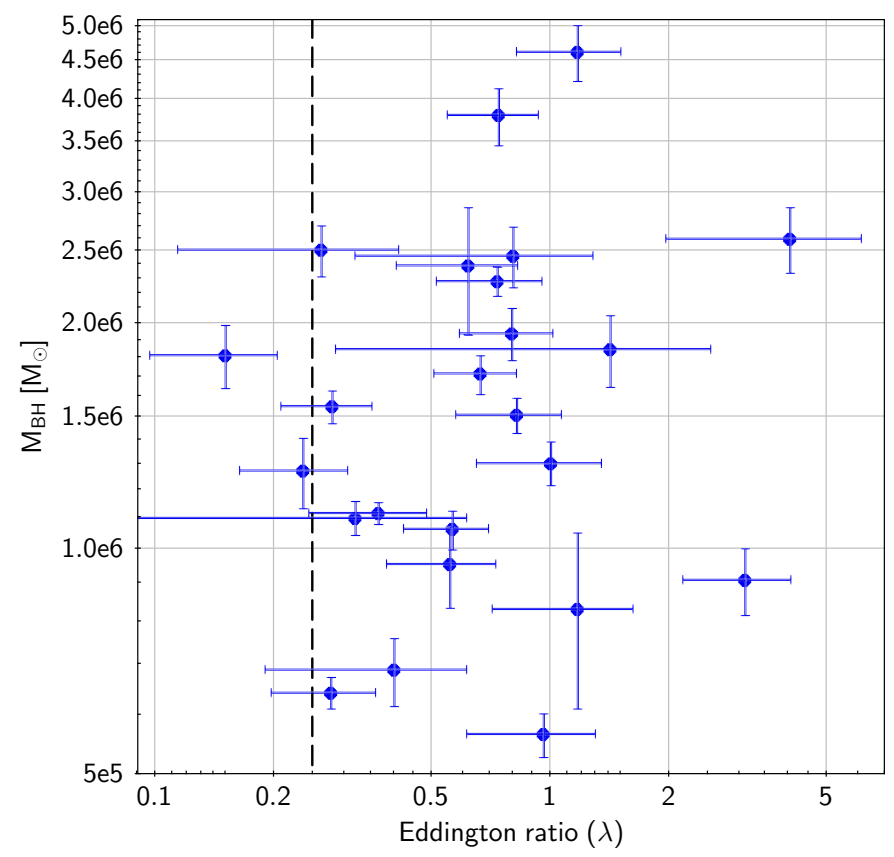

Fig. 8. $M_{\mathrm{BH}}$ and Eddington ratio for the NLS1. The dashed line represents the lower limit to the observed Eddington ratio for NLS1 $\left(\lambda_{\mathrm{NLS} 1, \lim }=0.25\right)$.

\subsection{Obscuration hypothesis}

In order to explain the optical dullness of the sources with obscuration, we need to consider complete obscuration of the central engine and/or the NLR. One possibility is that the nuclear absorber is not distributed in the torus-like geometry assumed for the classical model, and so obscuration may be caused by spherical Compton-thick gas clouds, covering almost $4 \pi$ at the central engine (Comastri et al. 2002; Civano et al. 2007), implying that ionising photons cannot escape from the nuclear source to produce the NLR. Or, if the covering of the nuclear source is not complete, only few photons can reach the NLR, leading to attenuated emission lines from this region. On the other hand, Rigby et al. (2006) proposed the presence of extranuclear dust and gas. These are distributed on large scales (i.e. dust lanes) in the host galaxy along our line of sight and which may hide the emission lines from the NLR.

According to the X-ray spectral fitting results (see Sect. 3.3), the hydrogen column density $N_{H}$ is, for almost all of the sources, much smaller than $10^{22} \mathrm{~cm}^{-2}$, corresponding to an optical extinction value $A_{V} \ll 4.5 \mathrm{mag}$ (assuming the Galactic relation: $A_{V}=4.5 \times 10^{-22} N_{\mathrm{H}}$ of Güver \& Özel 2009), and so are not heavily absorbed.

For AGN with column density greater than $>10^{24} \mathrm{~cm}^{-2}$, $\mathrm{X}$-rays are significantly absorbed and scattered above a few $\mathrm{keV}$, so X-ray spectra below $10 \mathrm{keV}$ do not provide information on the real column density. The possibility that some sources are Compton-thick can be investigated by evaluating the total absorption by comparing two indirect AGN luminosities. The $6 \mu \mathrm{m}$ continuum luminosity is a good proxy for the AGN luminosity, like the hard X-ray luminosity. We estimate the $6 \mu \mathrm{m}$ flux, and thus luminosity, from the WISE fluxes in the four bands (3.4 $\mu \mathrm{m}, 4.6 \mu \mathrm{m}, 12 \mu \mathrm{m}$ and $24 \mu \mathrm{m})$ using a weighted linear least square fitting (e.g. $\log f_{v} \propto \log \lambda$ ). This analysis, see Fig. 9, confirms that all of the sources lie above the Compton-thick region of the diagram (Goulding et al. 2011).

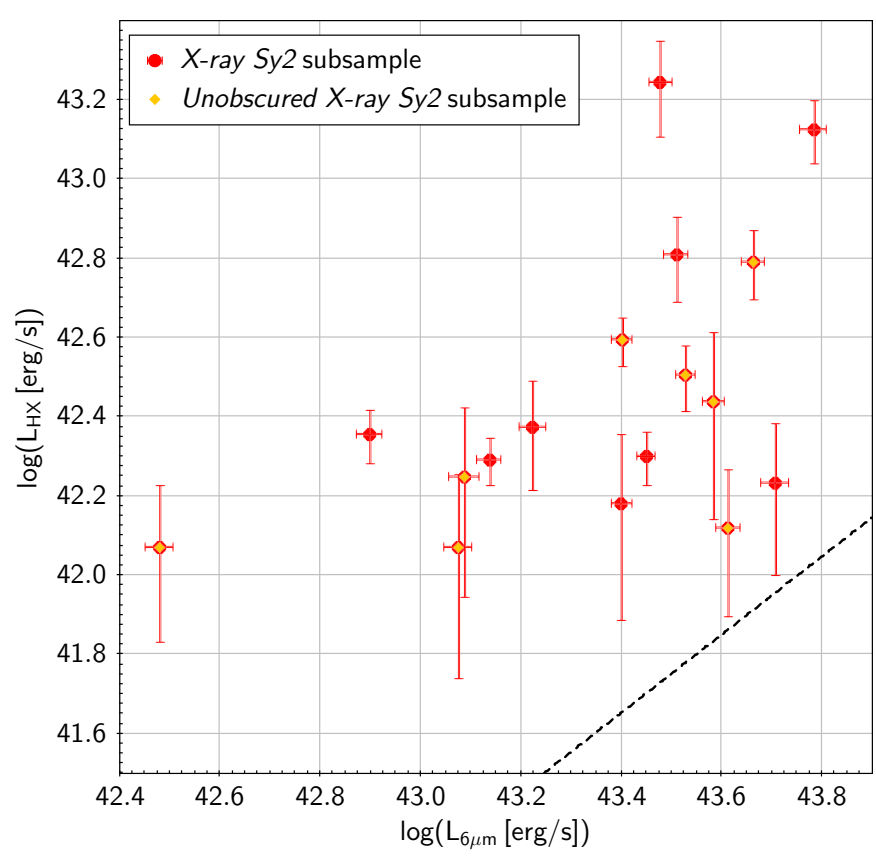

Fig. 9. Comparison of the $L_{\mathrm{HX}}$ and $L_{6} \mu \mathrm{m}$, two indirect AGN luminosity indicators, confirming the absence of Compton-thick sources. The Compton-thick region on the diagram lies below the black dashed line.

We can conclude that obscuration of the central $\mathrm{BH}$ is unlikely to be the cause of the elusiveness of optical AGN signatures in these sources. Moreover, the absence of observed obscuration in the sources with narrow emission lines (i.e. unobscured $\mathrm{X}$-ray Sy2) is not due to Compton-thick absorbers, which can be confirmed by comparing the [OIII] and hard X-ray luminosities. In the presence of high levels of absorption, the hard X-ray luminosity is expected to be depressed by an amount related to the absorbing column density, while the [OIII] emission, coming from the NLR, should be unaffected. Compton-thick sources $\left(N_{\mathrm{H}}>10^{24} \mathrm{~cm}^{-2}\right)$ are expected to have a thickness parameter $T=L_{\mathrm{HX}} / L_{\text {[OIII] }}$ smaller than 0.1 (i.e. $\log T<-1$ ) (Bassani et al. 1999). The X-ray Sy2 class in this sample have $\log T$ between 0.5 and 2.5 (see Fig. 2), so do not appear to be Compton-thick sources.

\subsection{Optical dilution hypothesis}

Another possible explanation for the absence of an AGN signature in the optical spectrum of these sources is the optical dilution of the AGN activity by starlight from the host galaxy, with a possible additional star-formation contribution.

The contribution of starlight from the host galaxy can be estimated using the $4000 \AA$ break $\left(\Delta_{n}\right)$ :

$\Delta_{\mathrm{n}}=\frac{F^{+}-F^{-}}{F^{+}}$

where $F^{+}$and $F^{-}$represent the mean value of the flux density in the rest-frame regions 4000-4100 $\AA$ and 3850-3950 $\AA$ respectively (definition from Balogh et al. 1999, less sensitive to reddening by dust). The nuclear emission is dominant if the value of $\Delta_{\mathrm{n}}$ is below $20 \%$, whilst the host galaxy light dominates the continuum if $\Delta_{\mathrm{n}}$ ranges from $20 \%$ to $60 \%$. For $\Delta_{\mathrm{n}}$ values between $20-40 \%$ both the AGN and the host galaxy contribute to the spectrum. 


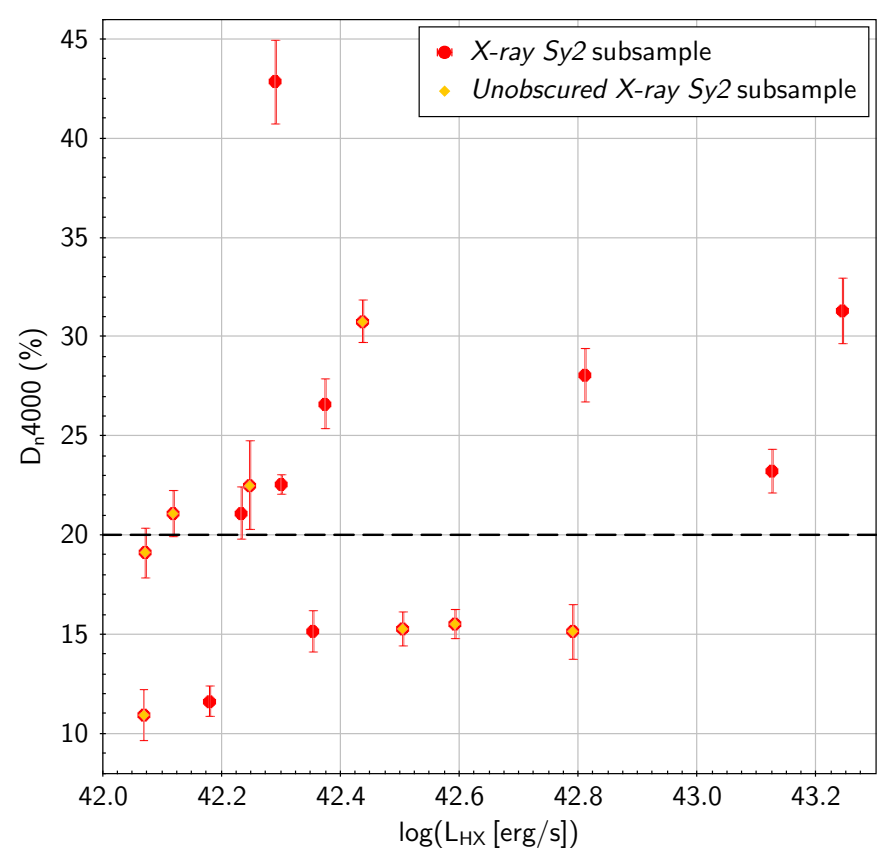

Fig. 10. $4000 \AA$ break $\left(\Delta_{\mathrm{n}}\right)$ values for the sources in the X-ray Sy2 group. The optical continuum of a galaxy is dominated by AGN for $\Delta_{\mathrm{n}}<20 \%$, and for larger values it is dominated by the host galaxy light.

We can see in Fig. 10 that 10 of the 17 X-ray Sy2 sources have their blue continuum dominated by the host galaxy starlight and so the AGN light is diluted.

Moreover, by comparing the observed $\mathrm{H} \alpha$ line luminosity with the value expected for an AGN (using the relation from Panessa et al. 2006), most of these sources have Balmer emission lines fluxes in excess, which could be due to star-formation. Any star-formation contribution will enhance the Balmer lines and so reduce the $[\mathrm{OIII}] / \mathrm{H} \beta$ and $[\mathrm{NII}] / \mathrm{H} \alpha$ line ratios, moving the sources from the AGN region to the SF region of the BPT diagram.

So intrinsic weakness of the AGN, in respect to the host galaxy, combined in some cases with a star-formation contribution could explain the elusiveness of about $60 \%$ of the sources of the X-ray Sy2 group.

\subsection{Low accretion rate hypothesis}

Finally, undiluted optically dull AGN could be characterised by intrinsically weak optical emission from the accretion disk. In the case of low accretion rates, AGN are expected to be optically underluminous compared with typical AGN due to radiatively inefficient accretion flows (RIAFs, Yuan \& Narayan 2004).

The Eddington ratio $\left(\lambda=L_{\mathrm{bol}} / L_{\mathrm{Edd}}\right)$ of these sources is computed using the Eqs. (2) and (3) described in the Sect. 3.5 for the bolometric luminosity. The $\mathrm{BH}$ masses of the Sy2 sources were estimated using the relation between $M_{\mathrm{BH}}$ and the rest-frame $K$-band bulge luminosity (from Graham 2007; as improved by Trump et al. 2011):

$\log \left(\frac{M_{\mathrm{BH}}}{M_{\odot}}\right)=0.93 \cdot\left(\log \left(L_{K, \text { bulge }}\right)-0.3 z\right)+32.30$

with $L_{K \text {,bulge }}$ in $\mathrm{erg} \mathrm{s}^{-1}$ and $L_{K \text {,bulge }}=0.5 \cdot L_{K \text {,host }}$ assumed for narrow-line AGN.

As can be seen in Fig. 11, the Sy2 sources have low Eddington ratios, $0.001<\lambda<0.05$, compared to typical AGN

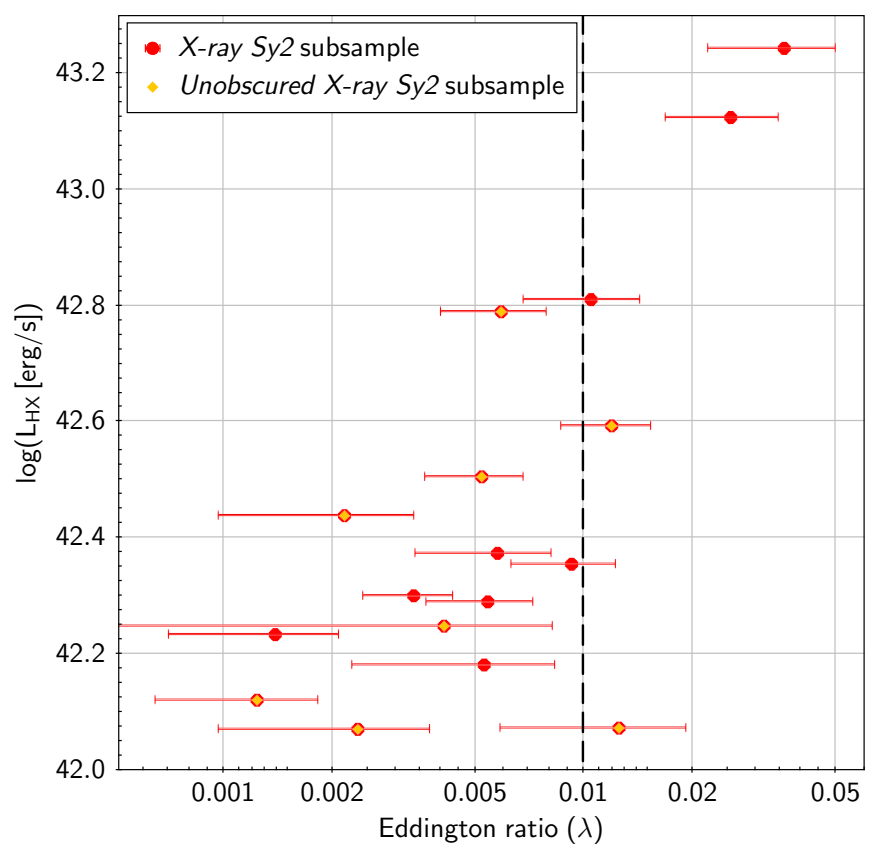

Fig. 11. Hard X-ray luminosity vs. Eddington ratio $(\lambda)$ of the X-ray Sy2 sources. The values of $\lambda$ are low compared to typical AGN and the low HR2 sources (in yellow) have very low accretion rates $\left(\lambda<10^{-2}\right.$; black dash line) and so are good candidates to be true Sy2.

( $\lambda \sim 0.07-1$, Heckman et al. 2004), providing an explanation for the optical elusiveness of these sources. Moreover, all the unobscured X-ray Sy2 sources have very low accretion rates $\left(\lambda<10^{-2}\right)$. A recent study by Trump et al. (2011) showed that for a specific accretion rates $\left(\lambda<10^{-2}\right)$, the BLR disappears because of the occurrence of a RIAF extending to the inner region of the accretion disk. Below this specific accretion rate, the disk wind, which is supposed to form in the BLR, is no longer supported. The resulting AGN are unobscured but lack broad emission lines in their optical spectrum: they are called true Sy2.

Therefore, the 8 unobscured X-ray Sy 2 sources are good candidates to be true Sy2 AGN.

\subsection{True Sy2 candidates: expected FWHM and luminosity of the broad lines}

True Sy 2 candidates have no intrinsic absorption so, according to the Unified Model, are expected to exhibit broad optical emission lines. To confirm their classification as true Sy2, we can compute their expected BL FWHM and luminosity and compare these with the observed values.

For the broad $\mathrm{H} \alpha$ line, the FWHM is directly related to the BH mass and continuum luminosity at $5100 \AA$ (see Eq. (1), Sect. 3.5).

The predicted broad $\mathrm{H} \alpha$ luminosity can be estimated from the empirical relation of Greene \& Ho (2005):

$L_{\mathrm{Broad} \mathrm{H} \alpha}=(5.25 \pm 0.02) \times 10^{42}\left(\frac{\lambda L_{5100}}{10^{44} \mathrm{erg} \mathrm{s}^{-1}}\right)^{(1.157 \pm 0.005)} \mathrm{erg} \mathrm{s}^{-1}$

The rms scatter in this relation is quite small, being $\sim 0.2$ dex.

The observed flux and line width of $\mathrm{H} \alpha$, from the optical spectrum, and the predicted values for the broad component from the equations above and (1), are given Table 3.

The observed spectrum in the $\mathrm{H} \alpha$ region with the predicted broad $\mathrm{H} \alpha$ component superimposed is shown in Fig. 12. The 
E. Pons and M. G. Watson: A new sample of X-ray selected narrow emission-line galaxies. I.
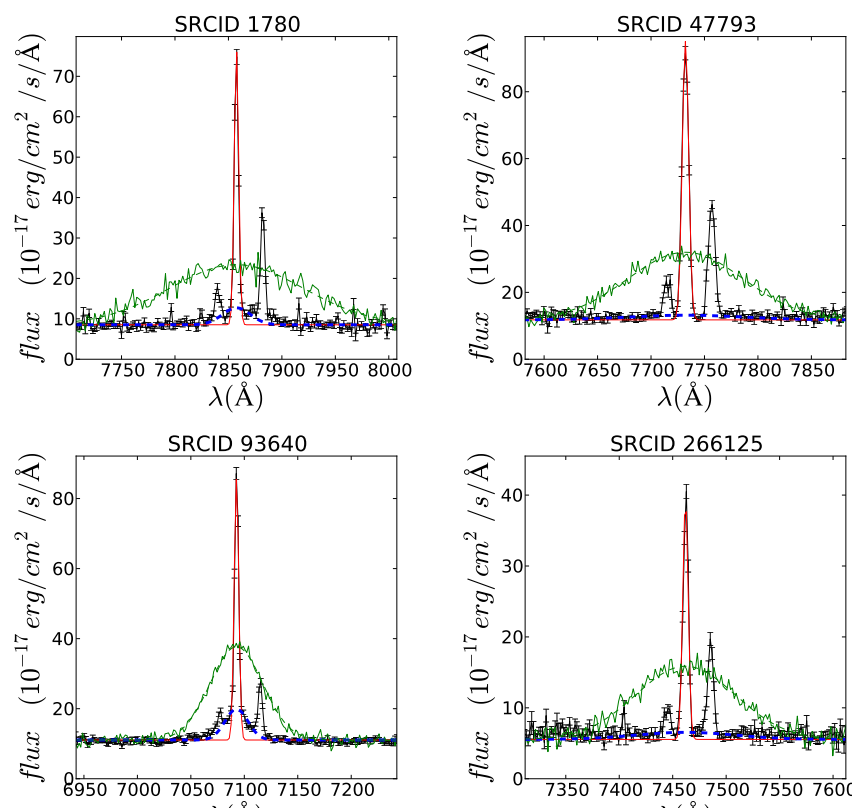

$\lambda(\AA)$

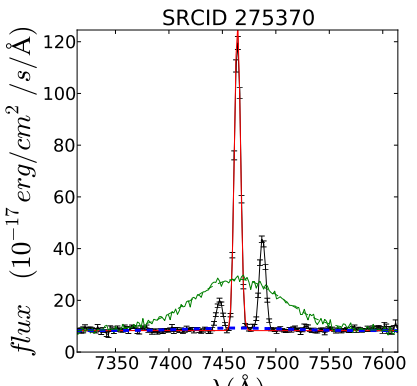

$\lambda(\AA)$

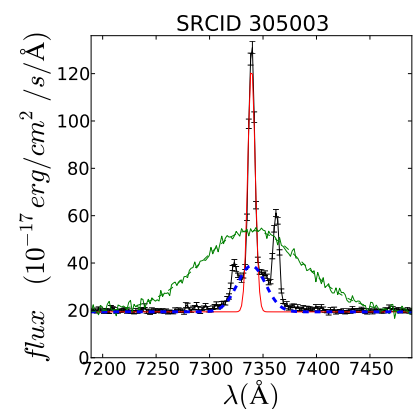

$\lambda(\AA)$

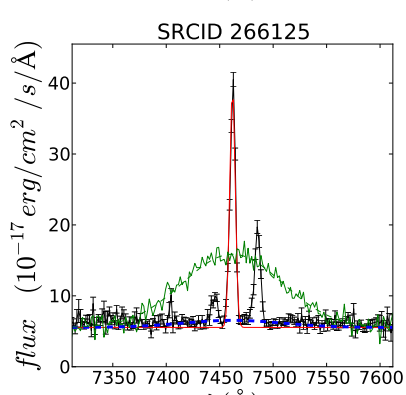

$\lambda(\AA)$

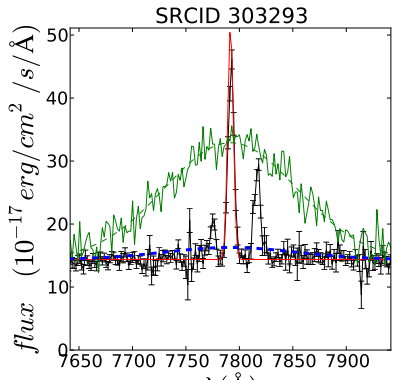

$\lambda(\AA)$

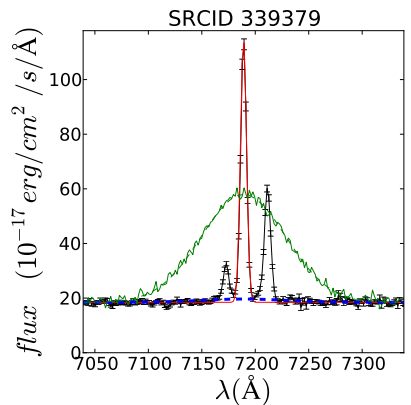

Fig. 12. Optical spectra of the true Sy 2 candidates around the $\mathrm{H} \alpha$ region. The observed $\mathrm{H} \alpha$ line is fitted with a Gaussian profile (red curve) while the predicted broad component is represented by a Gaussian (green). The dashed blue line represents the fitted weak broad line or the upper limit to the broad component.

$\mathrm{H} \alpha$ line is fitted with a Gaussian profile. The predicted broad component is modelled by Gaussian function with width and flux as discussed above; the predicted component is plotted with randomised measurement noise equivalent to that of the actual observed spectrum. The broad line flux plotted in Fig. 12 is the fitted value (SRCIDs 1780, 93640, 305003) where a possible weak line is detected or, if no line is detected, an upper limit, set to twice the apparent noise level (see Table 3, Col. 3).

We can then compare the predicted and measured parameters for $\mathrm{H} \alpha$. We can see Fig. 13 that there is a large difference between the predicted and measured (or upper limit) fluxes of the broad $\mathrm{H} \alpha$ component. For a consistent observed flux with the

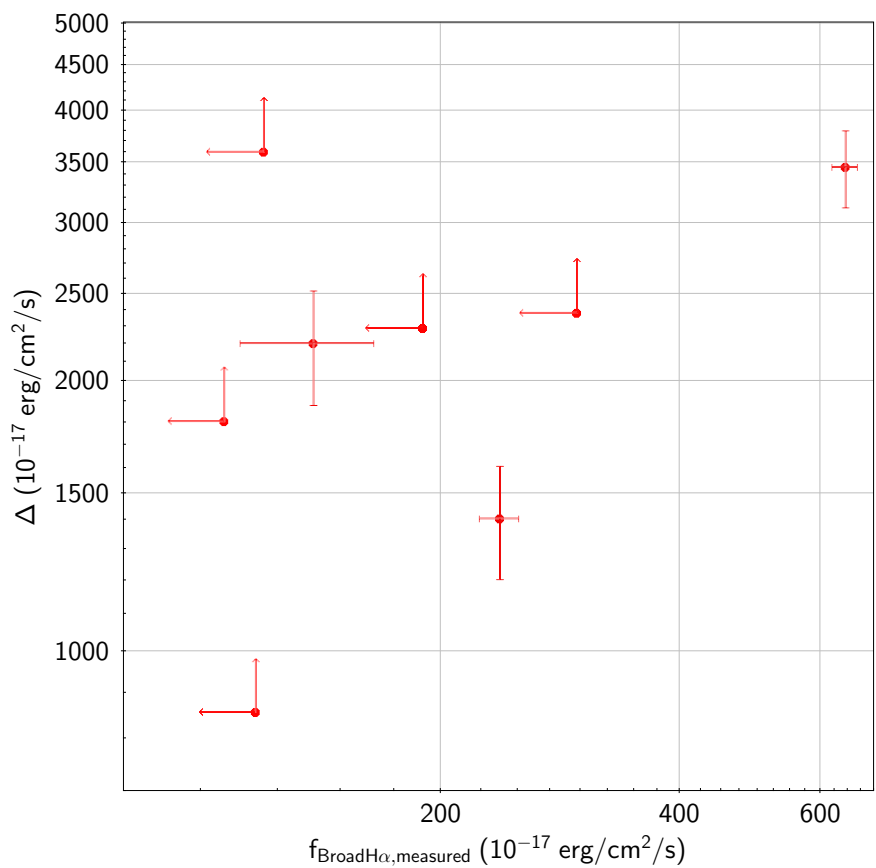

Fig. 13. Difference between the predicted and measured flux (noted as $\Delta$ ) vs. measured flux for the $\mathrm{H} \alpha$ broad component of the true Sy2 candidates.

prediction, we expect a value of $\Delta$ (with $\Delta=f_{\mathrm{BroadH} \alpha \text {,predicted }}$ $f_{\mathrm{BroadH} \alpha \text {,measured }}$ ) around 0 . However, we can see that the minimum $\Delta$, even if considering the errors, is about 1000 (with a mean value 2000 ), so far away from the expected value of 0 . Thus, there is no chance that the observed flux was consistent with the predicted value for the broad $\mathrm{H} \alpha$ line. The observed, or upper limit, broad component flux constitutes only 0.5 to $2.4 \%$ of the predicted flux. Moreover, these sources have an observed broad $\mathrm{H} \alpha$ flux $\gtrsim 1$ dex lower than the predicted value. While the scatter in the Greene \& Ho (2005) $L_{\mathrm{Broad} \alpha}-\lambda L_{5100}$ relation, due to the intrinsic scatter of QSO properties, is only $\sim 0.2 \mathrm{dex}$; so the observed difference between the observed and predicted fluxed is a real effect. We thus conclude that a broad $\mathrm{H} \alpha$ line would have been securely detected if present at the expected level. In conclusion these sources clearly lack broad lines and seem to be true Sy2.

\section{Summary and conclusions}

We have investigated the nature of a sample of X-ray selected AGN optically (mis)classified as SF galaxies in the BPT diagnostic diagram (elusive AGN), and have discussed possible reasons for the misclassification. For this purpose, we have looked at their optical and X-ray properties.

We find that $59 \%$ of the elusive AGN are very likely to be NLS1, showing a steep X-ray spectrum with no intrinsic absorption consistent with a low hardness ratio, forbidden line widths smaller than the Balmer line widths and a high Eddington ratio with low mass black hole. It thus seems that these sources are misclassified in the optical as SF galaxies because of the additional contribution to the Balmer line flux from the BLR and NLR; while the BPT diagram classification assumes contributions only from the NLR.

The other $41 \%$ of the remaining elusive AGN have the some properties of type $2 \mathrm{AGN}$, they have only narrow emission lines in their optical spectra, X-ray luminosity of an AGN but some 
Table 3. True Sy2: observed and predicted parameters for $\mathrm{H} \alpha$.

\begin{tabular}{c|cc|cc|cc}
\hline \hline \multirow{2}{*}{ SRCID } & \multicolumn{2}{|c|}{ Narrow $\mathrm{H}^{1}$} & \multicolumn{2}{c|}{${\text { Broad }{ }^{2}}^{2}$} & \multicolumn{2}{c}{${\text { Predicted broad } \mathrm{H}^{3}}^{a}$} \\
& flux $^{a}$ & $F W H M^{b}$ & flux $^{a}$ & $F W H M^{b, *}$ & flux $^{a}$ & $F W H M^{b}$ \\
\hline 1780 & $357 \pm 6$ & $188 \pm 3$ & $139 \pm 26$ & $1181 \pm 430$ & $2338 \pm 295$ & $5638 \pm 375$ \\
47793 & $631 \pm 8$ & $275 \pm 3$ & $<190$ & 4940 & $2741 \pm 264$ & $4940 \pm 286$ \\
93640 & $398 \pm 5$ & $212 \pm 2$ & $238 \pm 14$ & $1071 \pm 278$ & $1642 \pm 190$ & $2454 \pm 279$ \\
266125 & $216 \pm 4$ & $242 \pm 4$ & $<117$ & 4428 & $1210 \pm 238$ & $4428 \pm 1769$ \\
275370 & $843 \pm 9$ & $266 \pm 2$ & $<107$ & 4047 & $2130 \pm 221$ & $4047 \pm 246$ \\
303293 & $243 \pm 43$ & $237 \pm 16$ & $<296$ & 5638 & $2887 \pm 209$ & $5638 \pm 303$ \\
305003 & $887 \pm 9$ & $327 \pm 3$ & $644 \pm 23$ & $1265 \pm 268$ & $4099 \pm 318$ & $4579 \pm 177$ \\
339379 & $691 \pm 7$ & $280 \pm 2$ & $<119$ & 3915 & $3934 \pm 222$ & $3915 \pm 155$ \\
\hline
\end{tabular}

Notes. ${ }^{(1)}$ Parameters from the optical spectrum, obtained by the SDSS line fitting pipeline (SDSS SpecLine table) (Fig. 12 red curve). ${ }^{(2)}$ Fitted weak line (SRCIDs 1780, 93640, 305003) or the upper limit of the broad component (Fig. 12 dashed blue line). ${ }^{(3)}$ Predicted broad H $\alpha$ component computed using equations of Sect. 4.4 (Fig. 12 green curve). ${ }^{(a)}$ Line flux in units of $10^{-17} \mathrm{erg} / \mathrm{cm}^{2} / \mathrm{s} .{ }^{(b)}$ Full Width at Half Maximum in units of $\mathrm{km} \mathrm{s}^{-1} .{ }^{(*)}$ The FWHM of the broad component is the fitted value when a weak broad line is present, otherwise it is fixed at the predicted value (Col. 7 of the table).

of them show no sign of absorption (or little absorption), it is that why we call them X-ray Sy2. Three possible explanations for their optical elusiveness have been investigated. Heavy obscuration by gas and dust is not a likely origin of the misclassification. However, two other possibilities could explain the optical elusiveness of these sources. Firstly, $60 \%$ of them are optically diluted by starlight (so are weak AGN compared to the host galaxy) with a possible star- formation contribution in some cases. Secondly, the X-ray Sy2 sources also have low accretion rates and are thus optically underluminous due to an intrinsic weakness of the AGN.

Moreover, among these X-ray Sy2, we find a population of true Sy2, e.g. undiluted AGN but with a lack of broad emission lines in their optical spectra. The absence of broad lines has been checked by comparing the observed optical spectra with the predicted flux and width of the $\mathrm{H} \alpha$ broad component. According to some theoretical models, their very low accretion rates cause the disappearance of the BLR.

Acknowledgements. We would like to thank Andrew Blain and Gordon Stewart, together with the referee, Jonathan Trump, for their useful comments. This work uses data from observations obtained with XMM-Newton, an ESA science mission with instruments and contributions directly funded by ESA Member States and NASA. Data from the SDSS is also utilised.Funding for SDSS-III has been provided by the Alfred P. Sloan Foundation, the Participating Institutions, the National Science Foundation, and the US Department of Energy Office of Science. The SDSS-III web site is http://www.sdss3.org/. This publication also makes use of data products from the Wide-field Infrared Survey Explorer, which is a joint project of the University of California, Los Angeles, and the Jet Propulsion Laboratory/California Institute of Technology, funded by the National Aeronautics and Space Administration.

\section{References}

Antonucci, R. 1993, ARA\&A, 31, 473

Baldwin, J. A., Phillips, M. M., \& Terlevich, R. 1981, PASP, 93, 5

Balogh, M. L., Morris, S. L., Yee, H. K. C., Carlberg, R. G., \& Ellingson, E. 1999, ApJ, 527, 54

Barth, A. J., Ho, L. C., Rutledge, R. E., \& Sargent, W. L. W. 2004, ApJ, 607, 90 Bassani, L., Dadina, M., Maiolino, R., et al. 1999, ApJS, 121, 473

Bentz, M. C., Peterson, B. M., Netzer, H., Pogge, R. W., \& Vestergaard, M. 2009, ApJ, 697, 160

Bianchi, S., Panessa, F., Barcons, X., et al. 2012, MNRAS, 426, 3225

Boller, T., Brandt, W. N., \& Fink, H. 1996, A\&A, 305, 53

Botte, V., Ciroi, S., Rafanelli, P., \& Di Mille, F. 2004, AJ, 127, 3168

Brinchmann, J., Charlot, S., White, S. D. M., et al. 2004, MNRAS, 351, 1151

Caccianiga, A., Severgnini, P., Della Ceca, R., et al. 2007, A\&A, 470, 557

Caccianiga, A., Severgnini, P., Della Ceca, R., et al. 2008, A\&A, 477, 735

Cash, W. 1979, ApJ, 228, 939

Castelló-Mor, N., Barcons, X., Ballo, L., et al. 2012, A\&A, 544, A48

Civano, F., Mignoli, M., Comastri, A., et al. 2007, A\&A, 476, 1223

Comastri, A., Mignoli, M., Ciliegi, P., et al. 2002, ApJ, 571, 771
Crummy, J., Fabian, A. C., Gallo, L., \& Ross, R. R. 2006, MNRAS, 365, 1067

Elvis, M., Schreier, E. J., Tonry, J., Davis, M., \& Huchra, J. P. 1981, ApJ, 246, 20

Fabbiano, G. 1989, ARA\&A, 27, 87

Filippenko, A. V., \& Ho, L. C. 2003, ApJ, 588, L13

Gierliński, M., \& Done, C. 2004, MNRAS, 349, L7

Goulding, A. D., Alexander, D. M., Mullaney, J. R., et al. 2011, MNRAS, 411, 1231

Graham, A. W. 2007, MNRAS, 379, 711

Greene, J. E., \& Ho, L. C. 2005, ApJ, 630, 122

Güver, T., \& Özel, F. 2009, MNRAS, 400, 2050

Heckman, T. M., Kauffmann, G., Brinchmann, J., et al. 2004, ApJ, 613, 109

Kauffmann, G., Heckman, T. M., Tremonti, C., et al. 2003a, MNRAS, 346, 1055

Kauffmann, G., Heckman, T. M., White, S. D. M., et al. 2003b, MNRAS, 341, 33

Kewley, L. J., Dopita, M. A., Sutherland, R. S., Heisler, C. A., \& Trevena, J. 2001, ApJ, 556, 121

Kormendy, J., \& Richstone, D. 1995, ARA\&A, 33, 581

La Franca, F., Fiore, F., Vignali, C., et al. 2002, ApJ, 570, 100

Marconi, A., Risaliti, G., Gilli, R., et al. 2004, MNRAS, 351, 169

Moran, E. C., Filippenko, A. V., \& Chornock, R. 2002, ApJ, 579, L71

Mushotzky, R. 2004, in Supermassive Black Holes in the Distant Universe, ed. A. J. Barger, Astrophys. Space Sci. Lib., 308, 53

Nandra, K., George, I. M., Mushotzky, R. F., Turner, T. J., \& Yaqoob, T. 1997, ApJ, 476, 70

Netzer, H., \& Trakhtenbrot, B. 2007, ApJ, 654, 754

Osterbrock, D. E., \& Ferland, G. J. 2006, Astrophysics of gaseous nebulae and active galactic nuclei, 2nd edn. (Sausalito: University Science Books)

Osterbrock, D. E., \& Pogge, R. W. 1985, ApJ, 297, 166

Panessa, F., Bassani, L., Cappi, M., et al. 2006, A\&A, 455, 173

Pineau, F.-X., Motch, C., Carrera, F., et al. 2011, A\&A, 527, A126

Ranalli, P., Comastri, A., \& Setti, G. 2003, A\&A, 399, 39

Reines, A. E., Sivakoff, G. R., Johnson, K. E., \& Brogan, C. L. 2011, Nature, 470, 66

Rigby, J. R., Rieke, G. H., Donley, J. L., Alonso-Herrero, A., \& Pérez-González, P. G. 2006, ApJ, 645, 115

Rodríguez-Ardila, A., Binette, L., Pastoriza, M. G., \& Donzelli, C. J. 2000, ApJ, 538,581

Ross, R. R., Fabian, A. C., \& Mineshige, S. 1992, MNRAS, 258, 189

Schlegel, D. J., Finkbeiner, D. P., \& Davis, M. 1998, ApJ, 500, 525

Stasińska, G., Cid Fernandes, R., Mateus, A., Sodré, L., \& Asari, N. V. 2006, MNRAS, 371, 972

Tremonti, C. A., Heckman, T. M., Kauffmann, G., et al. 2004, ApJ, 613, 898

Trouille, L., \& Barger, A. J. 2010, ApJ, 722, 212

Trump, J. R., Impey, C. D., Taniguchi, Y., et al. 2009, ApJ, 706, 797

Trump, J. R., Impey, C. D., Kelly, B. C., et al. 2011, ApJ, 733, 60

Urry, C. M., \& Padovani, P. 1995, PASP, 107, 803

Vasudevan, R. V., \& Fabian, A. C. 2007, MNRAS, 381, 1235

Wang, J. X., Malhotra, S., Rhoads, J. E., \& Norman, C. A. 2004, ApJ, 612, L109

Watson, M. G., Schröder, A. C., Fyfe, D., et al. 2009, A\&A, 493, 339

Xiao, T., Barth, A. J., Greene, J. E., et al. 2011, ApJ, 739, 28

Yuan, F. 2007, in The Central Engine of Active Galactic Nuclei, eds. L. C. Ho, \& J.-W. Wang, ASP Conf. Ser., 373, 95

Yuan, F., \& Narayan, R. 2004, ApJ, 612, 724

Zoghbi, A., Fabian, A. C., \& Gallo, L. C. 2008, MNRAS, 391, 2003 